\title{
Model-Based Robust Control Design for Magnetostrictive Transducers Operating in Hysteretic and Nonlinear Regimes
}

\author{
James M. Nealis ${ }^{1}$ and Ralph C. Smith ${ }^{2}$ \\ Department of Mathematics \\ Center for Research in Scientific Computation \\ North Carolina State University \\ Raleigh, NC 27695
}

\begin{abstract}
This paper addresses the development of robust control designs for high performance smart material transducers operating in nonlinear and hysteretic regimes. While developed in the context of a magnetostrictive transducer used for high speed, high accuracy milling, the resulting model-based control techniques can be directly extended to systems utilizing piezoceramic or shape memory alloy compounds due to the unified nature of models used to quantify hysteresis and nonlinearities inherent to all of these materials. When developing models and corresponding inverse filters or compensators, significant emphasis is placed on the utilization of the material's physics to provide the accuracy and efficiency required for real-time implementation of resulting model-based control designs. In the material models, this is achieved by combining energy analysis with stochastic homogenization techniques whereas the efficiency of forward algorithms is combined with monotonicity properties of the material behavior to provide highly efficient inverse algorithms. These inverse filters are then incorporated in $\mathcal{H}_{2}$ and $\mathcal{H}_{\infty}$ theory to provide robust control algorithms capable of providing high accuracy tracking even though the actuators are operating in nonlinear and hysteretic regimes. Through numerical examples, it is illustrated that the robust designs incorporating inverse compensators can achieve the required tracking tolerance of $1-2 \mu \mathrm{m}$ for the motivating milling application whereas robust designs which treat the uncompensated hysteresis and nonlinearities as unmodeled disturbances cannot achieve design specifications.
\end{abstract}

Keywords: Robust control design, hysteresis, constitutive nonlinearities, inverse filter, magnetostrictive transducers

\footnotetext{
${ }^{1}$ Email: jmnealis@unity.ncsu.edu, Telephone: (919) 515-8968

2Email: rsmith@eos.ncsu.edu; Telephone: (919) 515-7552; Corresponding Author
} 


\section{Introduction}

A growing emphasis in advanced control system designs focuses on the use of multifunctional materials, such as piezoceramics, magnetostrictives, and shape memory alloys, in integrated transducers to improve control authority and performance, reduce weight, and minimize power requirements. For example, piezoceramic compounds have both actuator and sensor capabilities due to the direct and converse piezoelectric effects, microscale set point accuracy, and high frequency $(\mathrm{kHz})$ operating capabilities. This has led to their use in applications ranging from nanopositioning mechanisms in an atomic force microscope (AFM) [23, 24, 25, 26] to inertial sensors in an accelerometer [9]. Magnetostrictive materials also offer both actuator and sensor capabilities as well as broadband transduction and large output forces. Recent applications and products exploiting these properties include high accuracy, high speed milling devices [29, 38] and torque sensing for steering systems [5, 22]. Shape memory alloys (SMA) operate at lower frequencies $(<100 \mathrm{~Hz}$ for bulk materials) but offer the largest power densities of the three compounds. Hence they are being considered for applications ranging from vibration dissipation in buildings and flexible aerospace structures to active shape configuration of an airfoil to optimize flight characteristics while minimizing drag [19, 27].

Whereas all of these compounds offer significant and unique actuator and/or sensor capabilities, they also exhibit varying degrees of hysteresis and constitutive nonlinearities throughout their operating range and frequency, stress and temperature-dependencies in various drive regimes. In some cases, the hysteresis can be mitigated through the drive electronics - e.g., charge or current control for piezoceramics is appropriate in certain applications $[15,16,17]$ - or feedback loops in the transducer. However, noise-to-signal ratios in sensor data and diminishing characteristics of high pass filters precludes a sole reliance on feedback loops for broadband and high frequency transduction. Furthermore, because damping properties of the materials are proportional to the area of the hysteresis loop, it is often advantageous to maximize rather than minimize hysteresis in the transducer design. This necessitates the development of robust control designs which incorporate the hysteresis in a manner which achieves specified tracking, damping and general performance criteria while employing the transducer materials in the nonlinear regimes which provide them with their unique actuator and/or sensor capabilities.

In this paper, we develop a model-based robust control design in the context of a magnetostrictive transducer operating in nonlinear and hysteretic regimes with high accuracy, high speed tracking specifications. A motivating application entails the use of the magnetostrictive transducer to mill out-of-round automotive parts at speeds of $3000 \mathrm{rpm}$ and tracking tolerances of 1-2 $\mu \mathrm{m}$ as detailed in [29,38] and depicted in Figure 1a. During the milling process, the cutting tip starts from a position of rest, is moved adjacent to the material, and then must follow a prescribed periodic trajectory required to yield the final geometry. Hence the trajectory to be tracked has the form depicted in Figure 1b. The forces required to drive the cutting head are generated through the reorientation of magnetic moments in the Terfenol-D rod in response to a field $H(t)$ produced by a current $I(t)$ to the surrounding solenoid. The surrounding magnet provides the bias necessary to achieve bidirectional strains. As illustrated in Figure 2, the field-magnetization and field-strain relations for the transducer exhibit significant hysteresis and constitutive nonlinearities which must be accommodated in models and model-based control designs.

Both the material behavior and control issues associated with this application are representative of those encounted when alternatively considering piezoceramic or shape memory alloy transducers and hence this

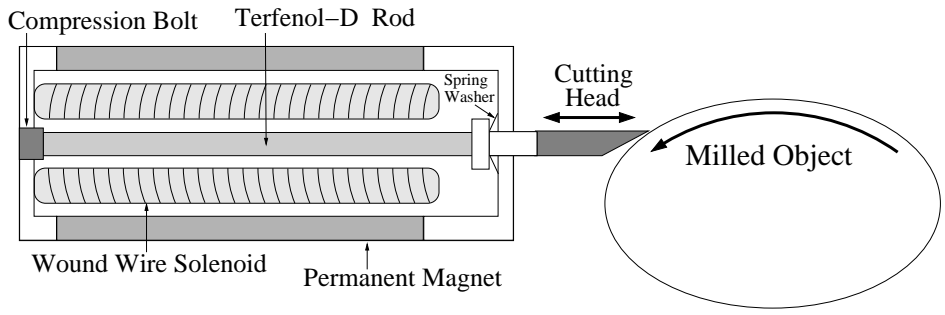

(a)

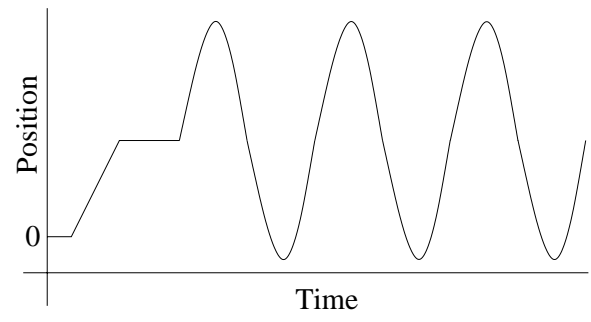

(b)

Figure 1: (a) Prototypical magnetostrictive transducer used for high speed, high accuracy milling. (b) Trajectory to be tracked by the cutting head. 


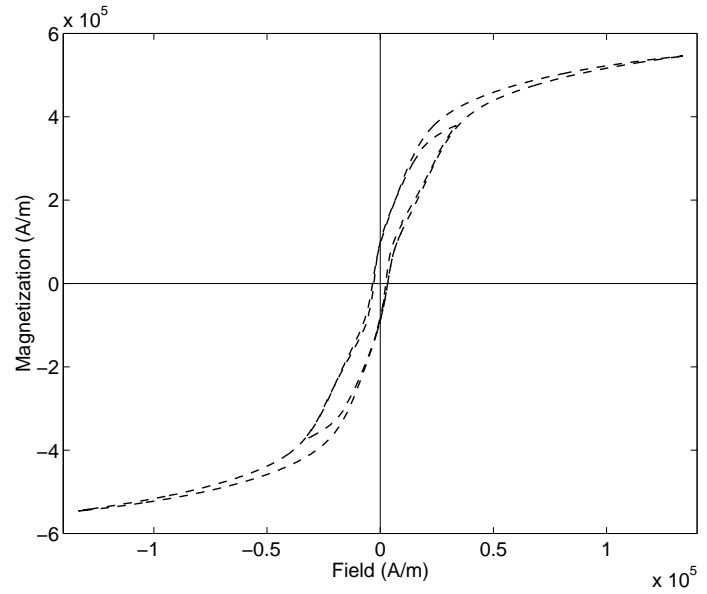

(a)

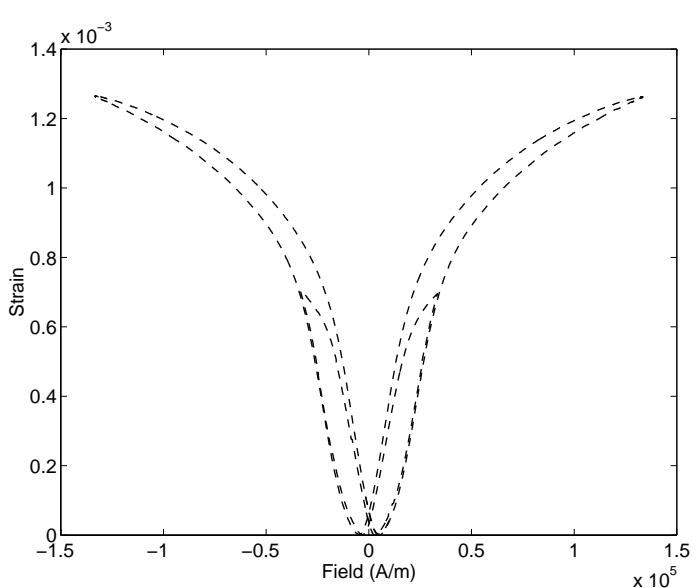

(b)

Figure 2: Hysteretic data measured in a Terfenol-D transducer as reported in [6]: (a) field-magnetization relation, and (b) field-strain relation.

transducer provides a framework for addressing robust control design for a broad range of multifunctional materials while clarifying the development by focusing on a specific application. It is detailed in $[33,35,36]$ that the common domain and ferroic nature of ferroelectric, ferromagnetic and ferroelastic materials can be exploited to develop unified models for the compounds and we employ one such model in the robust control design developed here. Hence the material behavior shown in Figure 2 is representative of that exhibited by a broad range of compounds and is characterized by unified models appropriate for all of these materials. Furthermore, the high accuracy specifications for the motivating milling application are representative of those encountered in a range of smart material control applications.

From the perspective of control design, the hysteresis inherent to the compounds introduces phase delays whereas saturation behavior introduces additional nonlinearities. These combined nonlinear effects produce disturbances which, if left unattenuated, produce unacceptable degradation in tracking performance. Sensor noise also produces disturbances which degrade the efficacy of state estimates and subsequent control inputs. We address both effects when constructing robust control designs for magnetostrictive transducers.

One strategy for designing robust control laws for hysteretic actuators is to employ nonlinear constitutive models to characterize the disturbances or perturbations $d$ which must be accommodated by control designs. This can reduce overly conservative robustness margins constructed solely through characterization experiments. A second strategy is to employ the models to construct inverse representations or compensators which can be employed as filters before the hysteretic actuator in the manner depicted in Figure 3. While such compensation is never exact due to discretization and modeling errors, the mismatch between inverse and hysteretic device can be designed to be small thus significantly reducing the disturbance $d$ associated with the transducer. It is this latter strategy that we consider here.

The use of model-based inverse representations for piezoceramic, magnetostrictive and shape memory compounds is not new and has received substantial attention in the context of adaptive, classical, and optimal

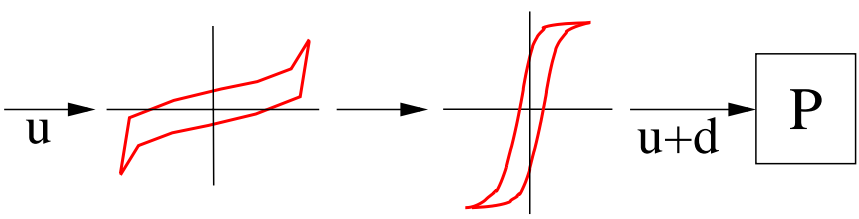

Figure 3: Approximate model inverse employed as a filter for robust control design in hysteretic and nonlinear systems. 
(LQR) control designs $[3,4,10,11,21,28,39,40]$. The primary emphasis has focused on Preisach representations (e.g., see $[39,40]$ ), due to their rigorous mathematical foundation and invertibility, as well as domain models $[21,28]$. While both modeling frameworks can be unified to quantify hysteresis in general ferroic compounds, both suffer disadvantages which degrade subsequent control performance in a number of applications. The Preisach methodology must be extended to accommodate the noncongruency, temperature-dependent and reversible behavior exhibited by magnetic (and most ferroic) materials whereas the domain-based theory of $[13,18,32,34]$ requires extensive modifications to guarantee the closure of minor loops in state feedback regimes. Furthermore, there is a growing emphasis on the design of control algorithms which accommodate strongly transient and quickly changing dynamics and are robust with respect to a variety of disturbances. This motivates the development of robust control designs utilizing inverse representations based on the modeling framework developed in $[30,31]$ for ferromagnetic materials with analogous models for piezoceramic compounds [37], shape memory alloys [19, 27], and unified ferroic compounds [35, 36].

We consider both $\mathcal{H}_{2}$ and $\mathcal{H}_{\infty}$ designs since the disturbances can be interpreted in a variety of manners. Since the $\mathcal{H}_{2}$ norm quantifies the maximum amplitude in the output due to bounded energy inputs, it provides a natural measure for sensor noise as well as disturbances due to both approximately compensated or uncompensated hysteresis. The variability due to uncompensated or partially compensated hysteresis and constitutive nonlinearities can also be viewed as a structural uncertainty in the actuator model which motivates consideration of the $\mathcal{H}_{\infty}$ norm.

The emphasis in this paper focuses on the incorporation of physics through energy-based techniques to provide control designs capable of achieving stringent tracking and speed criteria using advanced transducer designs rather than the analytical development of new control theories. Secondly, we emphasize the development of model-based control designs having the capability for real-time implementation at the speeds dictated by eventual applications. We rely on previous $\mathcal{H}_{2}$ and $\mathcal{H}_{\infty}$ theory - e.g., see $[1,8,20,41,42]$ - to provide a baseline for comparison in the absence of inverse compensation and a starting point from which to initiate robust control designs that incorporate the physical mechanisms which produce hysteresis through inverse compensators.

In Section 2, we summarize the development of nonlinear constitutive relations which quantify the inherent hysteresis, an algorithm for constructing inverse relations, and the system models used to characterize the magnetostrictive transducer depicted in Figure 1. We provide a system representation in Section 3 along with appropriate weighting filters and the $\mathcal{H}_{2}$ and $\mathcal{H}_{\infty}$ theory used to construct robust control algorithms. We illustrate the performance of these designs through numerical examples in Section 4. Specifically, we illustrate that for physically reasonable disturbance levels, designs based solely on $\mathcal{H}_{2}$ or $\mathcal{H}_{\infty}$ algorithms do not achieve specified control criteria whereas inclusion of the model-based inverse filters improves performance to the point where tracking criteria are achieved.

\section{Transducer Model}

The key to constructing an inverse compensator which accommodates hysteresis and constitutive nonlinearities is the development of material models which can be efficiently inverted. In this section, we consider the construction of models which quantify magnetostrictive transducer properties in two steps: (i) the construction of constitutive models which incorporate inherent hysteresis and material nonlinearities, and (ii) the employment of these constitutive models to quantify transducer outputs due to specified current inputs. This provides a framework for developing the model inverses employed in the robust control design in Section 3.

\subsection{Nonlinear Constitutive Relations}

The magnetic and magnetomechanical constitutive models we employ are based on the theory developed in $[30,31]$ and we summarize here those aspects relevant to the construction of magnetostrictive transducer models. For this discussion, we make the assumptions that temperatures are fixed, that eddy current losses are minimal and that prestress levels are sufficiently large that stress anisotropies dominate crystalline anisotropies. The assumption of fixed temperature is valid for magnetostrictive transducers utilizing water cooling whereas low frequency operating regimes or laminated rod designs reduce eddy current losses. Finally, the prestress levels which optimize performance are typically sufficient to dominate crystalline anisotropies [2] so the final assumption is valid for present transducer designs. 
To construct appropriate Helmholtz and Gibbs energy relations, we consider first the case in which applied stresses are either absent or negligible. Under the assumption that dipoles orient either with the applied field or diametrically opposite to it, which is valid for operating regimes in which stress anisotropies dominate crystalline anisotropies, and fixed temperature regimes, it is illustrated in [31] that a reasonable form of the Helmholtz energy is

$$
\psi(M)= \begin{cases}\frac{1}{2} \eta\left(M+M_{R}\right)^{2} & , M \leq-M_{I} \\ \frac{1}{2} \eta\left(M-M_{R}\right)^{2} & , M \geq M_{I} \\ \frac{1}{2} \eta\left(M_{I}-M_{R}\right)\left(\frac{M^{2}}{M_{I}}-M_{R}\right) & ,|M|<M_{I} .\end{cases}
$$

As depicted in Figure 4, $M_{R}$ and $M_{I}$ respectively denote the point at which the minimum of $\psi$ occurs and the inflection point. The point $M_{R}$ is also the local remanence magnetization at the domain level. Finally, the fact that $\eta$ is the reciprocal of the slope in the $H-M$ relation after switching can be utilized to determine initial parameter values when establishing the model for a given piezoceramic compound and application.

In the absence of applied stresses $\sigma$, a suitable magnetic Gibbs energy relation, which incorporates the work due to an applied field $H$, is

$$
\mathcal{G}=\psi-H M
$$

Note that the magnetostatic energy is $\mathcal{E}=\mu_{0} H M$ where $\mu_{0}$ denotes the magnetic permeability. The Gibbs relation (2) can thus be interpreted as incorporating $\mu_{0}$ into $\psi$ for simplicity.

To incorporate magnetoelastic coupling, we employ the extended Helmholtz relation

$$
\psi_{e}(M, \varepsilon)=\psi(M)+\frac{1}{2} Y^{M} \varepsilon^{2}-Y^{M} \zeta \varepsilon M^{2}
$$

and corresponding Gibbs energy

$$
\mathcal{G}(H, M, \varepsilon)=\psi(M)+\frac{1}{2} Y^{M} \varepsilon^{2}-Y^{M} \zeta \varepsilon M^{2}-H M-\sigma \varepsilon
$$

where $\psi$ is specified by (1) and $\varepsilon$ denotes longitudinal strains in the material. Here $Y^{M}$ denotes the Young's modulus at constant magnetization and $\zeta$ is a magnetoelastic coupling coefficient. We note that the second term in (3) incorporates the elastic energy. The quadratic dependence on the magnetization is motivated by characterization experiments [7] and can be theoretically justified in magnetostrictive transducers since prestress levels are such that stress anisotropies dominate crystalline anisotropies so that strains are due primarily to quadratic rotation processes.
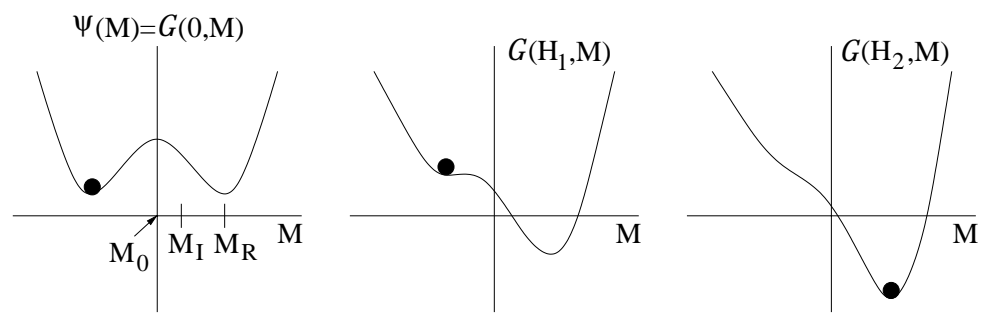

(a)
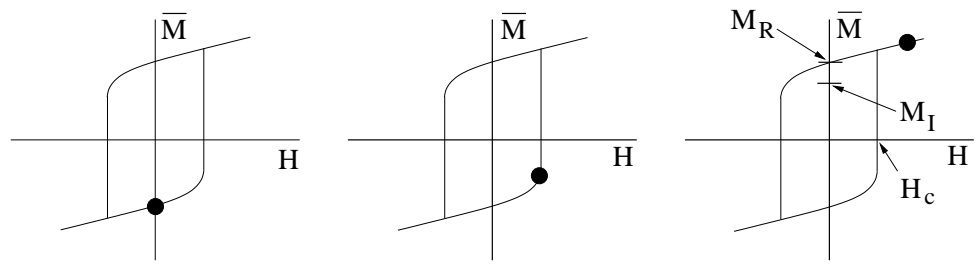

(b)

Figure 4: (a) Helmholtz energy $\psi$ and Gibbs energy $\mathcal{G}$ for $\sigma=0$ and increasing fields $H$. (b) Dependence of the local magnetization $\bar{M}$ on the field $H$ at the lattice level in the presence of thermal activation. 
Local average relations for the mesoscale magnetization are derived for two operating regimes: (i) conditions for which thermally activated relaxation processes are significant and, (ii) regimes for which relaxation processes are negligible. To incorporate relaxation processes, the Gibbs energy $\mathcal{G}$ is balanced with the relative thermal energy $k T / V$ over a lattice volume $V$ through the Boltzmann relation

$$
\mu(\mathcal{G})=C e^{-\mathcal{G} V / k T}
$$

which quantifies the probability $\mu$ of achieving the energy level $\mathcal{G}$. Here $C$ denotes a constant of integration which is constructed to have a value of unity when integrated over all admissible moment orientations, $k$ denotes Boltzmann's constant, and $T$ is the fixed temperature of operation. Under the assumption of two moment orientations, the local average magnetization is given by

$$
\bar{M}=x_{+}\left\langle M_{+}\right\rangle+x_{-}\left\langle M_{-}\right\rangle
$$

where $x_{+}$and $x_{-}$respectively denote the fraction of moments having positive and negative orientations and

$$
\left\langle M_{+}\right\rangle=\frac{\int_{M_{I}}^{\infty} M e^{-\mathcal{G}(H, M) V / k T} d M}{\int_{M_{I}}^{\infty} e^{-\mathcal{G}(H, M) V / k T} d M} \quad, \quad\left\langle M_{-}\right\rangle=\frac{\int_{-\infty}^{-M_{I}} M e^{-\mathcal{G}(H, M) V / k T} d M}{\int_{-\infty}^{-M_{I}} e^{-\mathcal{G}(H, M) V / k T} d M}
$$

are the expected magnetization values due to positively and negative oriented moments. The moment fractions satisfy the evolution equations

$$
\begin{aligned}
& \dot{x}_{+}=-p_{+-} x_{+}+p_{-+} x_{-} \\
& \dot{x}_{-}=-p_{-+} x_{-}+p_{+-} x_{+}
\end{aligned}
$$

where

$$
p_{+-}=\sqrt{\frac{k T}{2 \pi m}} \frac{e^{-\mathcal{G}\left(H, M_{I}\right) V / k T}}{\int_{M_{I}}^{\infty} e^{-\mathcal{G}(H, M) V / k T} d M} \quad, \quad p_{-+}=\sqrt{\frac{k T}{2 \pi m}} \frac{e^{-\mathcal{G}\left(H,-M_{I}\right) V / k T}}{\int_{-\infty}^{-M_{I}} e^{-\mathcal{G}(H, M) V / k T} d M}
$$

are the likelihoods of switching from positive to negative orientations and conversely. Here $m$ is the mass of lattice volume $V$. As depicted in Figure 4, the relation between the applied field $H$ and the local average magnetization $\bar{M}$ exhibits both hysteresis and nonlinear transition because the local magnetization (6) is probabilistic. The steepness of the transition depends on the ratio of $\mathcal{G}$ to $k T / V$.

For operating regimes in which thermally activated relaxation processes are negligible, the relative thermal energy $k T / V$ can be ignored and local average magnetization values $\bar{M}$ are computed directly from the necessary condition $\frac{\partial \mathcal{G}}{\partial M}=0$, or equivalently $H=\frac{\partial \psi_{e}}{\partial M}$. This yields the linear relation

$$
\bar{M}=\frac{H}{\eta-2 Y^{M} \zeta \varepsilon}+\Delta \frac{M_{R} \eta}{\eta-2 Y^{M} \zeta \varepsilon}
$$

which illustrates that $\eta$ denotes the reciprocal slope after switching in the absence of strains, $M_{R}$ is a local remanence value as depicted in Figure 4, and $\Delta$ indicates the moment orientations. To specify $\Delta$ and hence $\bar{M}$, we employ the Preisach notation

$$
\left[\bar{M}\left(H, \varepsilon ; H_{c}, \xi\right)\right](t)= \begin{cases}{\left[\bar{M}\left(H, \varepsilon ; H_{c}, \xi\right)\right](0)} & , \tau(t)=\emptyset \\ \frac{H}{\eta-2 Y^{M} \zeta \varepsilon}-\frac{M_{R} \eta}{\eta-2 Y^{M} \zeta \varepsilon} & , \tau(t) \neq \emptyset \text { and } H(\max \tau(t))=-H_{c} \\ \frac{H}{\eta-2 Y^{M} \zeta \varepsilon}+\frac{M_{R} \eta}{\eta-2 Y^{M} \zeta \varepsilon} & , \tau(t) \neq \emptyset \text { and } H(\max \tau(t))=H_{c}\end{cases}
$$

where $H_{c}=\eta\left(M_{R}-M_{I}\right)$, the transition times are specified by

$$
\tau(t)=\left\{t \in\left(0, T_{f}\right] \mid H(t)=-H_{c} \text { or } H(t)=H_{c}\right\}
$$

and the initial moment orientation is

$$
\left[\bar{M}\left(H, \varepsilon ; H_{c}, \xi\right)\right](0)= \begin{cases}\frac{H}{\eta-2 Y^{M} \zeta \varepsilon}-\frac{M_{R} \eta}{\eta-2 Y^{M} \zeta \varepsilon} & , H(0) \leq-H_{c} \\ \xi & ,-H_{c}<H(0)<H_{c} \\ \frac{H}{\eta-2 Y^{M} \zeta \varepsilon}+\frac{M_{R} \eta}{\eta-2 Y^{M} \zeta \varepsilon} & , H(0) \geq H_{c} .\end{cases}
$$

Here $\xi$ denotes the initial magnetization of the points with field levels between $-H_{c}$ and $H_{c}$. 

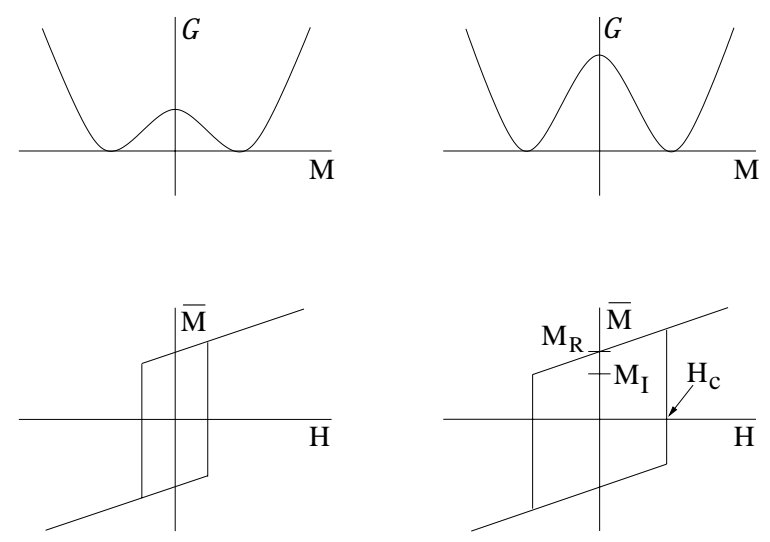

Figure 5: Free energy variations for a nonhomogeneous domain structure and the resulting variations in the local coercive field $H_{c}$ of the hysteresis kernel.

The relations (6), (7) and (8) are derived under the assumption that the lattice structure is homogeneous and hence the domain structure is uniform. This implies that free energy profiles for different regions of the Terfenol-D are identical. This assumption is not valid for general materials since it ignores material defects, polycrystallinity, lattice nonhomogeneities and nonuniform effective fields. To accommodate these properties, stochastic distributions are incorporated to develop a bulk magnetization model for nonhomogeneous Terfenol-D samples with nonconstant effective fields. Details regarding this development are provided in $[30,31]$.

To incorporate lattice nonhomogeneities due to material defects and variations, as well as nonuniformities due to polycrystallinity, we first consider the local coercive field $H_{c}=\eta\left(M_{R}-M_{I}\right)$ to be manifestations of an underlying distribution rather than constant (e.g., see Figure 5). Two a priori choices for densities are the normal function

$$
f\left(H_{c}\right)=C_{1} e^{-\left(H_{c}-\bar{H}_{c}\right)^{2} / b}
$$

with $H_{c}$ restricted to be positive, or the lognormal density

$$
f\left(H_{c}\right)=C_{1} e^{-\left[\ln \left(H_{c} / \bar{H}_{c}\right) / 2 b\right]^{2}}
$$

where $b, C_{1}$ and $\bar{H}_{c}$ are positive parameters. For the examples which follow, we employ (9) and refer the reader to [30] for details concerning (10).

Secondly, we incorporate variations in the effective field $H_{e}$ at the lattice level. As detailed in [12, 13], the applied field $H$ is augmented by induced fields of the form $H_{c}=\alpha M$ to yield general effective fields $H_{e}=H+\alpha M$. We assume here that these effective fields are normally distributed about the applied field as quantified by the density

$$
\widetilde{f}\left(H_{e}\right)=C_{2} e^{-\left(H_{e}-H\right)^{2} / \bar{b}} .
$$

The bulk magnetization model is then

$$
M(H)=C \int_{0}^{\infty} \int_{-\infty}^{\infty} \bar{M}\left(H_{e}+H ; H_{c}, \xi\right) e^{-H_{e}^{2} / \bar{b}} e^{-\left(H_{c}-\bar{H}_{c}\right)^{2} / b} d H_{e} d H_{c}
$$

where the kernel $\bar{M}$ is specified by (6), (7) or (8) and $C=C_{1} C_{2}$ is a positive constant which must be estimated through a least squares fit for a given transducer material.

The elastic constitutive relation is determined from the equilibrium condition

$$
\frac{\partial \mathcal{G}}{\partial \varepsilon}=0
$$

where $\mathcal{G}$ is specified in (4). This yields the coupled nonlinear constitutive relations

$$
\begin{aligned}
& \sigma=Y^{M} \varepsilon-Y^{M} \zeta M^{2} \\
& M(H, \varepsilon)=C \int_{0}^{\infty} \int_{-\infty}^{\infty} \bar{M}\left(H_{e}+H, \varepsilon ; H_{c}, \xi\right) e^{-H_{e}^{2} / \bar{b}} e^{-\left(H_{c}-\bar{H}_{c}\right)^{2} / b} d H_{e} d H_{c}
\end{aligned}
$$


for undamped magnetostrictive materials. These relations quantify the hysteresis and material nonlinearities illustrated in Figure 2 but neglect eddy current losses - hence they should be employed for laminated transducers or operation regimes in which eddy current losses are minimal. For the constant, high prestress conditions typical for present transducer designs, very adequate characterization is obtained under the simplifying assumption that $\varepsilon=0$ in the magnetization relation (12) or (13). Finally, the manner through which internal damping is incorporated is illustrated in Section 2.3 .

For numerical and experimental implementation of the magnetization model, the integrals must be approximated in an efficient manner. This can be accomplished by exploiting the decay exhibited by the kernels to truncate the domains followed by approximation using composite Gauss-Legendre quadrature formulae. If we denote abscissas by $H_{e_{j}}$ and $H_{c_{i}}$, and quadrature weights by $v_{i}, w_{j}$, this yields the discretized constitutive relations

$$
\begin{aligned}
& \sigma=Y^{M} \varepsilon-Y^{M} \zeta M^{2} \\
& M(H)=C \sum_{i=1}^{N_{i}} \sum_{j=1}^{N_{j}} \bar{M}\left(H_{e_{j}}+H ; H_{c_{i}}, \xi_{i}\right) e^{-H_{e_{j}}^{2} / \bar{b}} e^{-\left(H_{c_{i}}-\bar{H}_{c}\right)^{2} / b} v_{i} w_{j} .
\end{aligned}
$$

In all subsequent examples, we employ the linear kernel (7) or (8) for $\bar{M}$. Details regarding highly efficient algorithms for numerically implementing the discretized magnetization model are provided in [30] and analogous algorithms for piezoceramic compounds can be found in [37].

\section{Example - Model Validation}

To illustrate properties of the model, we summarize the manner through which it characterizes the quasistatic data plotted in Figure 2. Due to the very low frequency $(1 \mathrm{~Hz})$ at which data was collected, adequate approximations to measured strains are provided by the magnetostrictive component $\lambda=Y^{M} \zeta M^{2}$ of (14). We also employ $\varepsilon=0$ in the magnetization relation.

As detailed in [31], a least squares fit to the high drive level data yielded the parameters $\bar{H}_{c}=300 \mathrm{~A} / \mathrm{m}$, $M_{R}=3.7 \times 10^{4} \mathrm{~A} / \mathrm{m}, \eta=14, b=1 \times 10^{8} \mathrm{~A}^{2} / \mathrm{m}^{2}, \bar{b}=8 \times 10^{8} \mathrm{~A}^{2} / \mathrm{m}^{2}, C=2.52 \times 10^{-8}$ and $\zeta=$ $4.5 \times 10^{-15} \mathrm{~m}^{2} / \mathrm{A}^{2}$. The resulting model was then used to predict the moderate drive relations yielding the fits plotted in Figure 6. It is observed that both the fits and predictions are sufficiently accurate for subsequent model-based control design. Details regarding the experimental conditions can be found in $[6,7]$ whereas details regarding the model implementation as well as additional examples illustrating properties of the model, including closure of biased minor loops under quasistatic conditions, are provided in [31].

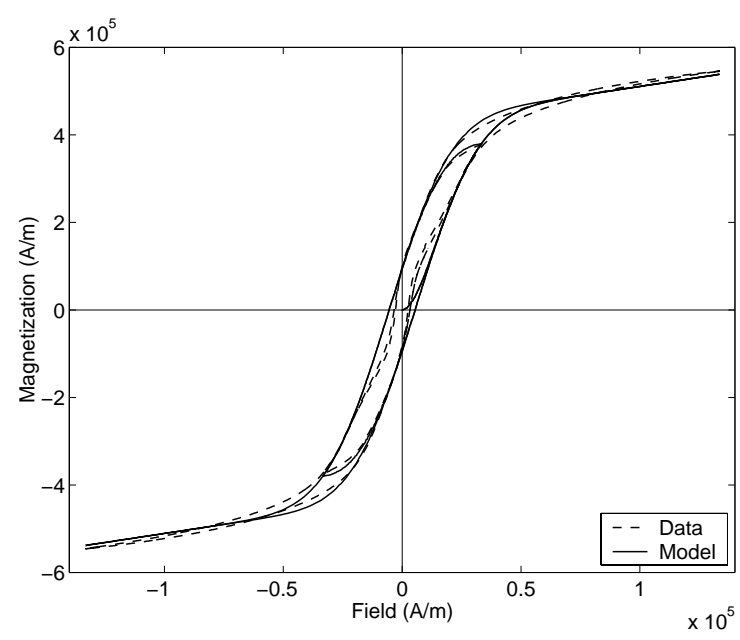

(a)

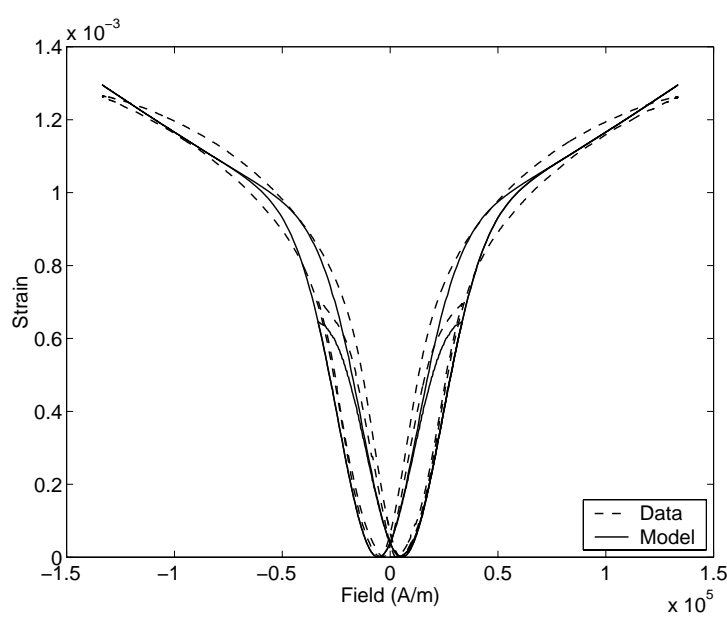

(b)

Figure 6: Experimental data (---) from [6] and model response (-): (a) field-magnetization relation, and (b) field-strain relation. 


\subsection{Model Inverse}

The efficiency of the forward model (14) is combined with the monotonic relation between $H$ and $M$ to construct inverse models which specify the field required to achieve a given magnetization. This inverse model is subsequently employed in the robust control designs detailed in Section 3 to achieve the stringent tracking criteria dictated by the application.

The construction of a model quantifying the inverse map between $M$ and $H$ is outlined in Algorithm 1 . For given values of $M_{\text {prev }}$ and $M_{\text {new }}$, the relation (14) is used to increment the field until the predicted magnetization $M_{t m p}$ has advanced beyond $M_{n e w}$, and the final field value $E_{\text {new }}$ is determined through linear interpolation between the final two predicted field values. When implementing the algorithm, the stepsize $\Delta H$ can be adaptively updated to ensure that the efficiency of the inverse algorithm is close to that of the forward algorithm.

\section{Algorithm 1.}

$$
\begin{aligned}
& \text { Specify } H_{\text {prev }}, M_{\text {prev }}, M_{\text {new }} \\
& \text { Specify } \Delta H \\
& d M=M_{\text {new }}-M_{\text {prev }} \\
& H_{t m p}=H_{\text {prev }}, M_{t m p}=M_{\text {prev }} \\
& \text { while } \operatorname{sgn}(d M)\left(M_{\text {new }}-M_{t m p}\right) \geq 0 \\
& \quad H_{t m p}=H_{t m p}+\Delta H d M \\
& \quad M_{t m p} \text { given by }(14) \\
& \text { end } \\
& H_{\text {new }} \text { given by linear interpolation }
\end{aligned}
$$

\section{Example - Inverse Compensation}

To illustrate the performance of the model inverse as well as errors or disturbances $d$ which must be accommodated by robust control designs when inverse compensators are applied as filters in the manner depicted in Figure 3, we applied a $1 \mathrm{~Hz}$ sinusoidal signal having a magnitude of $4 \times 10^{5} \mathrm{~A} / \mathrm{m}$ to the inverse model quantified by Algorithm 1. The output from the inverse model was subsequently applied to the forward model (14) and the resulting magnetization was compared with the original input signal. For stepsizes of $\Delta H=2$, this yielded the absolute and relative errors $d$ plotted in Figure 7. At this discretization level, it is observed that the linearization is highly accurate yielding relative errors under $1 \%$.

The computational speed of the inverse compensator depends on the size of the step taken when advancing the forward model with larger steps increasing the speed while decreasing the accuracy. The speed requirements

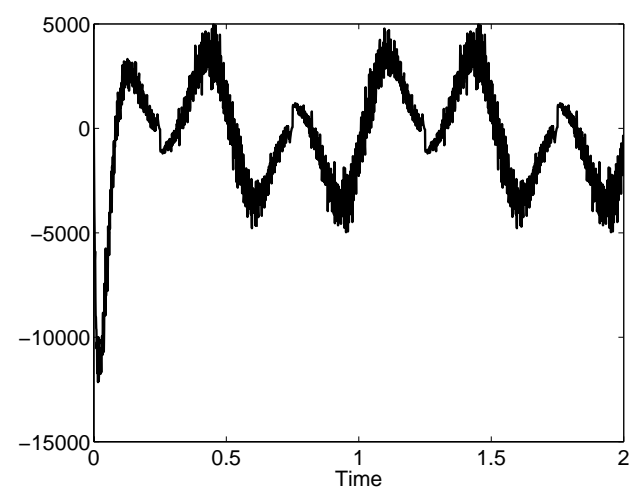

(a)

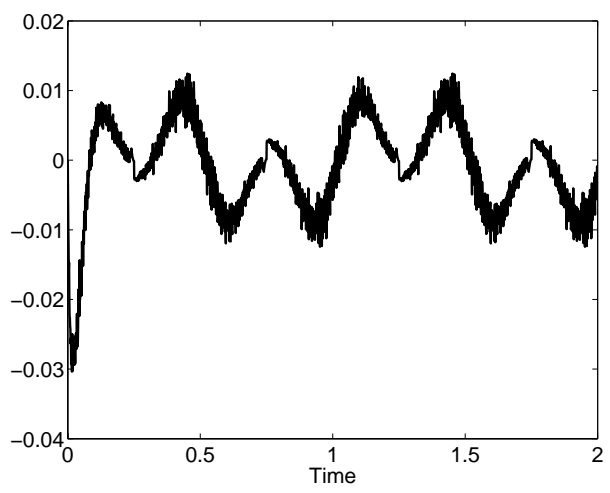

(b)

Figure 7: (a) Disturbance $d$ in the approximate inversion process depicted in Figure 3, and (b) relative disturbance $d /\left(4 \times 10^{5}\right)$. 
of real-time control implementation may necessitate relative large steps which increases the uncompensated disturbance in the plant which must be accommodated by the robust control design. However, the disturbances due to discretization errors accrued with small sample rates are still significantly less than those due to uncompensated hysteresis and constitutive nonlinearities which can be on the order $10^{5} \mathrm{~A} / \mathrm{m}$. As illustrated in the examples of Section 4, this permits the design of robust control algorithms utilizing approximate inverse filters which achieve specified tracking criteria whereas designs which treat the uncompensated hysteresis and nonlinearities solely as disturbances fail to meet these criteria.

\subsection{Transducer Model}

The constitutive model (14) quantifies the linear relation between stresses and strains as well as the nonlinear and hysteretic dependence of the stress on input fields $H$ through the magnetization model. Hence it characterizes the local elastic behavior of the Terfenol-D rod in a magnetostrictive transducer but it does not incorporate spatial dependence or internal damping. In this section, we employ the constitutive relation (14) to develop system models which quantify the displacements and forces generated by the magnetostrictive transducer in response to input currents $I(t)$. We summarize first the PDE model developed in [7] as well as the ODE system obtained through a finite element discretization. For transducer designs in which flux shaping is utilized to minimize end effects, it is illustrated that the second-order ODE system can be adequately approximated by a second order scalar differential equation to facilitate control design.

We assume that the left end of the Terfenol-D $\operatorname{rod}(x=0)$ is fixed whereas the right end $(x=L)$ is constrained by a damped oscillator and has an attached point mass $M_{L}$, as depicted in Figure 8, to model general loads encountered in applications. Furthermore, we assume that operation is biased about the point $\left(H_{0}, M_{0}\right)$ through the permanent magnet to achieve bi-directional strains. The Young's modulus, Kelvin-Voigt damping coefficient, and density are respectively denoted $Y^{M}, c_{D}$ and $\rho$. The constraining spring has stiffness $k_{L}$ and damping coefficient $c_{L}$. We let $w$ denote the longitudinal rod displacements therefore the strains are given by $\varepsilon=\frac{\partial w}{\partial x}$.

The direct use of the constitutive relation (13) or (14) yields an undamped model for the Terfenol-D rod. To incorporate Kelvin-Voigt damping, we posit that stress is actually proportional to a linear combination of strain, strain rate and squared magnetization to obtain

$$
\sigma(t, x)=Y^{M} \frac{\partial w}{\partial x}(t, x)+c_{D} \frac{\partial^{2} w}{\partial x \partial t}(t, x)-Y^{M} \zeta M^{2}(t, x)
$$

for $0 \leq x \leq L$. We note that the relation (15) is identical to the relation obtained in [7] if the magnetoelastic coupling coefficient is defined as $\zeta=\lambda_{s} / M_{s}$ with $\lambda_{s}$ and $M_{s}$ denoting the saturation magnetostriction and saturation magnetization, respectively.

As detailed in [7], the balancing of forces yields

$$
\rho A \frac{\partial^{2} w}{\partial t^{2}}=\frac{\partial N_{t o t}}{\partial x}
$$

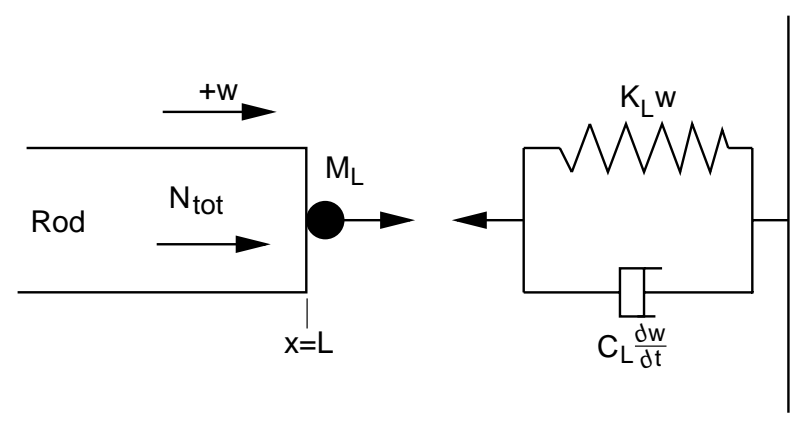

Figure 8: Spring, damped oscillator, and point mass used to model loads in applications. 
where $A$ is the cross sectional area of the Terfenol-D rod and the force resultant is specified by

$$
N_{t o t}(t, x)=Y^{M} A \frac{\partial w}{\partial w}(t, x)+c_{D} A \frac{\partial^{2} w}{\partial x \partial t}(t, x)-Y^{M} A \zeta M(t, x)^{2} .
$$

To obtain appropriate boundary conditions, we first note that $w(t, 0)=0$. Balancing forces at $x=L$ gives

$$
N_{t o t}(t, L)=-k_{L} w(t, L)-c_{L} \frac{\partial w}{\partial t}(t, L)-M_{L} \frac{\partial^{2} w}{\partial x \partial t}(t, L) .
$$

Initial conditions are taken to be $w(0, x)=0$ and $\frac{\partial w}{\partial x}(0, x)=0$.

As detailed in [7], formulation of the model (16) in weak form and spatial discretization utilizing linear finite elements yields the vector ODE system

$$
\begin{aligned}
& \mathcal{M} \ddot{\vec{y}}(t)+\mathcal{C} \dot{\vec{y}}(t)+\mathcal{K} \vec{y}(t)=\zeta \mathcal{B}\left[M^{2}(H)\right](t) \\
& \vec{y}(0)=\vec{y}_{0}, \quad \dot{\vec{y}}(0)=\vec{y}_{1}
\end{aligned}
$$

where $\vec{y}(t) \in \mathcal{R}^{N}$ is the state vector, $\mathcal{M} \in \mathcal{R}^{N \times N}, \mathcal{C} \in \mathcal{R}^{N \times N}, \mathcal{K} \in \mathcal{R}^{N \times N}$ respectively denote the mass, damping and stiffness matrices, and $\mathcal{B} \in \mathcal{R}^{N}$ contains integrated basis functions resulting from the inputs. For general systems, large $N$ (e.g., $N=32$ ) may be required to achieve convergence. This may limit the speed at which model-based control designs can be implemented which, for certain high speed applications, motivates consideration of an approximating lumped parameter model.

For transducer design in which the permanent magnet is constructed to minimize end effects, measurements with a Hall probe illustrate that nearly uniform fields

$$
H(t, x)=n I(t)
$$

are achieved along the length of the rod. Here $n$ denotes the number of coils per unit length in the solenoid and $I(t)$ is the current applied to the solenoid. In such a case, each finite element section of the rod reacts identically to the uniform magnetic field which motivates the consideration of the lumped scalar model

$$
\begin{aligned}
& m \ddot{y}(t)+c \dot{y}(t)+k y(t)=\varsigma\left[M^{2}(H)\right](t) \\
& y(0)=y_{0} \quad \dot{y}(0)=y_{1}
\end{aligned}
$$

where $y$ is the tip displacement. The scalars $k, c$ and $\varsigma$ are determined by a fit to the Galerkin approximation (18) or data from the physical device. The convergence properties of the model (18) and the accuracy of the scalar model (20) for transducers with uniform flux paths are illustrated in Figure 9.

Finally, experiments indicate that for moderate drive levels about a bias $\left(H_{0}, M_{0}\right)$, stresses exhibit an approximately linear dependence on the magnetization but a nonlinear and hysteretic dependence on the field

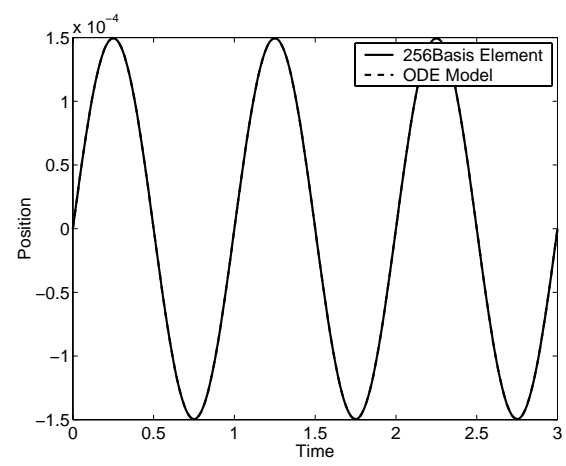

(a)

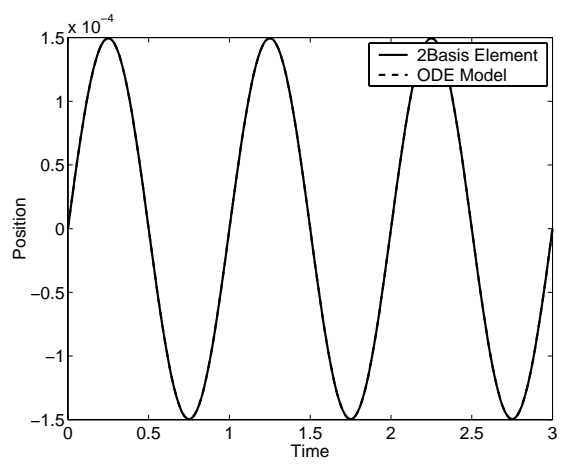

(b)

Figure 9: (a) Comparison of the finite element model (18) with a bias and the scalar model (21) with (a) $N=2$, and (b) $N=256$ basis elements. 
$H$ or current $I$ to the solenoid. This observation motivates us to linearize about the bias level $M_{0}$ to obtain the final model

$$
\begin{aligned}
& \ddot{y}(t)+\widehat{c} \dot{y}(t)+\widehat{k} y(t)=\widehat{\varsigma}[M(H)](t) \\
& y(0)=y_{0} \quad \dot{y}(0)=y_{1} .
\end{aligned}
$$

A model fit to the Terfenol-D data plotted in Figure 2 yields the parameter values $\widehat{c}=7.8899 \times 10^{3}, \widehat{k}=$ $6.4251 \times 10^{7}$ and $\widehat{\varsigma}=1.3724 \times 10^{-2}$. We note that while the model (21) exhibits a linear dependence on $M$, it retains the fully hysteretic and nonlinear dependence on $H$ and $I$ through the relations (12) and (19).

\section{Robust Control Design}

The construction of an inverse representation for the hysteresis and constitutive nonlinearities inherent to magnetostrictive transducers can significantly reduce unmodeled plant disturbances when applied as a filter before the physical device as depicted in Figure 3. However, this strategy does not completely eliminate the disturbance due to modeling and discretization errors thus yielding an input error $d$. Furthermore, the presence of ubiquitous sensor noise will diminish the accuracy of state estimates and subsequent control inputs. To provide a control framework which achieves the specified tracking tolerance of 1-2 $\mu \mathrm{m}$ in the presence of these disturbances, we construct $\mathcal{H}_{2}$ and $\mathcal{H}_{\infty}$ algorithms which incorporate the model inverse developed in Section 2. This includes a detailed discussion of the weighting filters employed in the design since they play a crucial role in achieving the desired accuracy. Numerical examples illustrating the performance of the $\mathcal{H}_{2}$ and $\mathcal{H}_{\infty}$ designs are provided in Section 4.

\subsection{System Representation}

The physical control system consists of the magnetostrictive transducer depicted in Figure 1a, and the control objective is to track a reference trajectory $r$ which prescribes the position of the cutting head throughout the milling procedure. During this process, the cutting head starts at rest, is moved adjacent to the material where it is held for a specified amount of time, and then is driven periodically to mill the out-of-round object. This yields a reference signal $r$ of the form depicted in Figure 1b. During the periodic cutting process, the head must maintain a tolerance of 1-2 $\mu \mathrm{m}$.

For control design, we employ the model (21) which specifies the displacement of the Terfenol-D rod tip in response to an applied field $H(t)$ or current $I(t)$ - see (19). We designate the ODE model as the plant $P$ in the system representation depicted in Figure 10a and let $d$ denote errors in the plant input due either to unmodeled

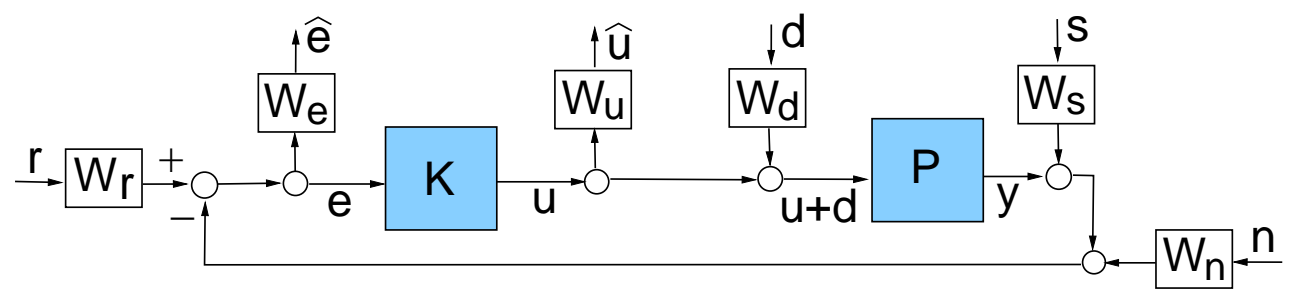

(a)

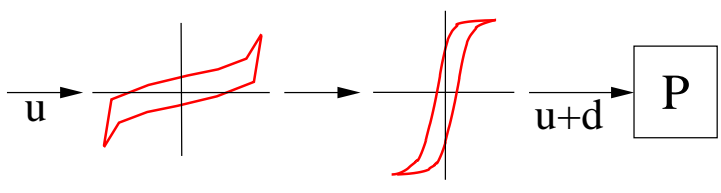

(b)

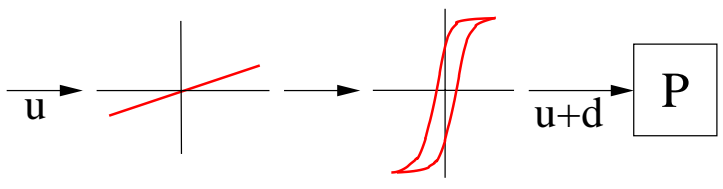

(c)

Figure 10: (a) System representation including input disturbances $d$ and sensor noise $n$ and $s$ in the transducer. (b) Disturbance $d$ due to inverse filtering errors. (c) Disturbance $d$ due to scaled but unmodeled hysteresis and constitutive nonlinearities. 
hysteresis and constitutive nonlinearities or approximation errors accrued when applying the inverse filters, as depicted respectively in Figure $10 \mathrm{~b}$ and Figure 10c. Noise in the measurement of $y$ is separated into two signals, $s$ and $n$. We assume a $60 \mathrm{~Hz}$ noise signal, due to the sensing apparatus, which is represented by $s$. The signal $n$ represents higher frequency noise which may be attributed to the sensing device or other external disturbances. These two signals are separated since we wish to weight them independently. The output signals $\widehat{e}$ and $\widehat{u}$ denote the weighted tracking error and weighted output of the controller $K$, respectively. Finally, $W_{u}$, $W_{d}, W_{e}, W_{r}, W_{s}$ and $W_{n}$ are weighting functions chosen to maximize the performance of the controller utilizing a priori knowledge of the characteristics of the corresponding signals in the manner detailed in Section 3.2 .

We first represent the open loop system. The maps from the inputs $r, d, s$ and $n$ to the outputs $e, \widehat{e}$ and $\widehat{u}$ are respectively given by

$$
\begin{aligned}
e & =W_{r}[r]-\left(P\left[W_{d}[d]+u\right]+W_{n}[n]+W_{s}[s]\right) \\
& =W_{r}[r]-P\left[W_{d}[d]\right]-W_{n}[n]-W_{s}[s]-P[u] \\
\widehat{e} & =W_{e}[e] \\
\widehat{u} & =W_{u}[u] .
\end{aligned}
$$

The transfer function matrix $G$ from the inputs $r, d, n, s$ and $u$ to the outputs $\widehat{e}, \widehat{u}$, and $e$ is then specified by

$$
G=\left[\begin{array}{ccccc}
W_{e} W_{r} & -W_{e} P W_{d} & -W_{e} W_{n} & -W_{e} W_{s} & -W_{e} P \\
0 & 0 & 0 & 0 & W_{u} \\
W_{r} & -P W_{d} & -W_{n} & -W_{s} & -P
\end{array}\right]
$$

To formulate the system representation in a manner which fulfills the assumptions of theorems guaranteeing the existence of optimal or sub-optimal controllers, we chose a class of weighting functions $W_{u}$ which have a nonzero $D$ matrix in the corresponding state space representation. Furthermore, the state space representation of either $W_{n}$ or $W_{s}$ is required to have a nonzero $D$ matrix and $W_{e}$ should satisfy $D=0$. For the weighting functions constructed in Section 3.2, the open loop system can be partitioned as

$$
G(s)=\left[\begin{array}{c|cc}
A & B_{1} & B_{2} \\
\hline C_{1} & 0 & D_{12} \\
C_{2} & D_{21} & 0
\end{array}\right]=\left[\begin{array}{cc}
G_{11} & G_{12} \\
G_{21} & G_{22}
\end{array}\right],
$$

where

$$
\begin{gathered}
G_{11}=\left[\begin{array}{c|c}
A & B_{1} \\
\hline C_{1} & 0
\end{array}\right], \quad G_{12}=\left[\begin{array}{c|c}
A & B_{2} \\
\hline C_{1} & D_{12}
\end{array}\right] \\
G_{21}=\left[\begin{array}{c|c}
A & B_{1} \\
\hline C_{2} & D_{21}
\end{array}\right], \quad G_{22}=\left[\begin{array}{c|c}
A & B_{2} \\
\hline C_{2} & 0
\end{array}\right]
\end{gathered}
$$

respectively represent the transfer functions from $w$ to $z, u$ to $z, w$ to $e$, and $u$ to $e$ in the linear fractional transformation (LTF) system representation depicted in Figure 11. Details regarding the construction of component matrices in state space realizations for transfer functions can be found in Section 3.5 of [41].

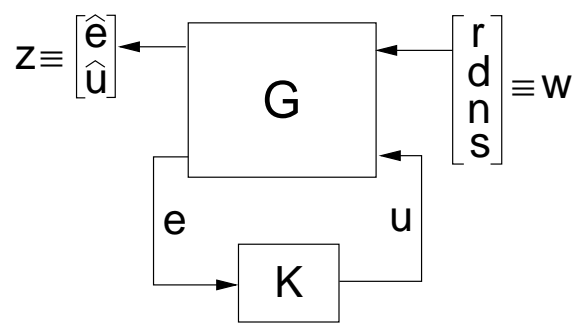

Figure 11: Linear fractional transformation (LFT) representation of the transducer model. 


\subsubsection{System Assumptions}

To guarantee the existence of unique control inputs for the $\mathcal{H}_{2}$ and $\mathcal{H}_{\infty}$ algorithms from [41] which are summarized in Section 3.3, we make the following assumptions regarding the system representation (23).

A1. $\left(A, B_{1}\right)$ is controllable and $\left(C_{1}, A\right)$ is observable

A2. $\left(A, B_{2}\right)$ is stabilizable and $\left(C_{2}, A\right)$ is detectable

A3. $D_{12}=\left[\begin{array}{l}0 \\ I\end{array}\right]$ and $D_{21}=\left[\begin{array}{ll}0 & I\end{array}\right]$

A4. $\left[\begin{array}{cc}A-j \omega I & B_{2} \\ C_{1} & D_{12}\end{array}\right]$ has full column rank for all $\omega$

A5. $\left[\begin{array}{cc}A-j \omega I & B_{1} \\ C_{2} & D_{21}\end{array}\right]$ has full row rank for all $\omega$

Assumption A1 is necessary to guarantee the existence of a stabilizing controller. Assumptions A4 and A5 along with A2 guarantee the existence of solutions to corresponding Riccati equations. Assumption A3 ensures the $\mathcal{H}_{2}$ and $\mathcal{H}_{\infty}$ problems are nonsingular. We note that if $D_{12}$ has full column rank and $D_{21}$ has full row rank but they do not satisfy Assumption A3, a normalizing procedure can be performed as described in [41].

While there are only two internal states in the transducer model (21), the inclusion of weighting filters can substantially increase the dimension of the plant thus motivating the removal of uncontrollable and unobservable states through the techniques detailed in [41] to obtain a minimal realization for the system. For the weighting filters described in Section 3.2, this leads to a reduction from 70 states to 31 states for the numerical examples presented in Section 4.

For the system under consideration, Assumptions A1 and A2 are automatically guaranteed for the minimal realization employed for control design. Since $D_{12}^{*} C_{1}=0$ for the considered system, Assumption A4 is equivalent to the condition that the open loop matrix $A$ has no purely imaginary eigenvalues which can be directly verified for the considered filter designs. If we let $R_{2}=D_{21} D_{21}^{*}$, then Assumption A5 is achieved if $A^{*}-C_{2}^{*} R_{2}^{-1} D_{21} B_{1}^{*}$ has no purely imaginary eigenvalues. This too has been validated for the filters constructed in Section 3.2 thus verifying that all of the assumptions are met for the transducer control system.

\subsection{Weighting Functions}

An important facet of robust control design focuses on the construction of the weighting functions $W_{u}, W_{d}$, $W_{e}, W_{r}, W_{s}$ and $W_{n}$. We summarize here techniques for constructing these filters for the motivating system having hysteretic and nonlinear inputs, and we refer the reader to [14, 41] for a more general discussion concerning the choice of weights.

As noted in Section 3.1, we consider sensor noise having a narrowband component $s$ at $60 \mathrm{~Hz}$ and a high frequency, broadband component $n$. To construct a narrowband filter which minimally weights frequencies above and below a specified bandwidth, one typically employs an $n$ th-order Chebyshev filter which is simply a system whose frequency response function satisfies

$$
\left|H_{c b}(\omega)\right|=\frac{1}{1+\epsilon_{p} C_{n}^{2}\left(\omega / \omega_{s}\right)}
$$

where $\omega_{s}$ is the sampling frequency, $\epsilon_{p}$ is a parameter that controls the speed of the rolloff, and the polynomials $C_{n}$ are the $n$ th-order Chebyshev polynomials defined by the recursion

$$
C_{0}(\omega)=1, \quad C_{1}(\omega)=\omega C_{0}(\omega) \quad C_{n+1}(\omega)=2 \omega C_{n}(\omega)-C_{n-1}(\omega) .
$$

To weight the $60 \mathrm{~Hz}$ noise $s$, we employ a sixth-order, bandpass Chebyshev filter $W_{s}$ having a bandwidth of $10 \mathrm{~Hz}$ centered at $60 \mathrm{~Hz}$. From the frequency response plotted in Figure 12a, it is observed that $W_{s}$ heavily 


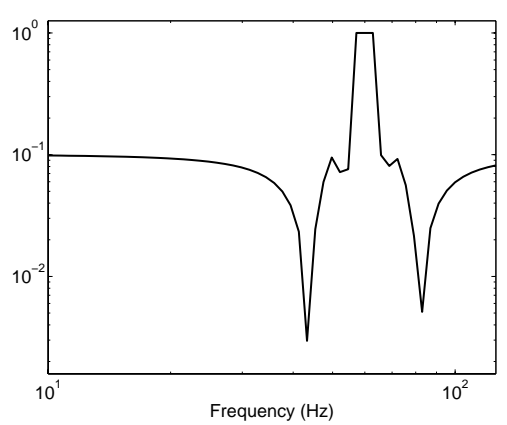

(a)

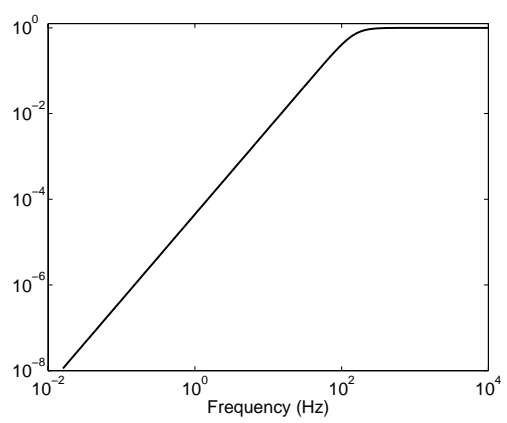

(b)

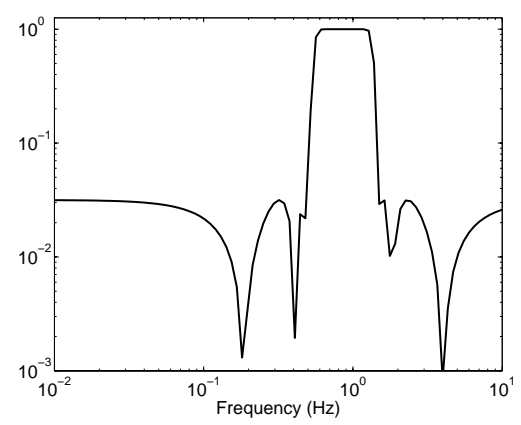

(c)

Figure 12: Frequency responses of (a) the narrowband Chebyshev filter $W_{s}$, (b) the high pass Butterworth filter $W_{n}$, and (c) the Chebyshev filter $W_{r}$.

weights frequencies in the neighborhood of $60 \mathrm{~Hz}$ with a sharp rolloff outside the specified bandwidth. We recall that either $W_{s}$ or $W_{n}$ is required to have a nonzero $D$ matrix in the state space realization and the filter $W_{s}$ is appended to force a nonzero $D$ to satisfy this requirement.

An $n$ th-order Butterworth filter having the frequency response

$$
\left|H_{b w}(\omega)\right|=\frac{1}{\sqrt{1+\left(\omega / \omega_{s}\right)^{2 n}}}
$$

provides a commonly employed highpass filter, where $\omega_{s}$ again denotes the sampling frequency. To weight the simulated high frequency noise $n$, we employed a fourth-order Butterworth filter having the frequency response plotted in Figure 12b.

The weight $W_{r}$ targets components of the reference signal which should be emphasized during tracking. For example, the accuracy of the initial ramp in the signal depicted in Figure 1b, which serves the purpose of bringing the cutting head adjacent to the material, is less crucial than maintaining a tolerance of 1-2 $\mu \mathrm{m}$ during the milling phase of the process. As detailed in Section 4, the properties of the control design can be illustrated by considering a $1 \mathrm{~Hz}$ periodic signal following the ramping up phase. The weight $W_{r}$ was specified to be the sixth-order passband Chebyshev filter plotted in Figure 12c having a bandwidth of $1 \mathrm{~Hz}$ and centered at $1 \mathrm{~Hz}$. If additional accuracy is desired during the ramping phase of the process, this weight can be modified to additionally emphasize lower frequency signals. Finally, one would simply change the center point when experimentally implementing the control law at the specified rate of $3000 \mathrm{rpm}$.

We consider two constructs for $W_{d}$ corresponding to the two techniques depicted in Figure 10 for accommodating the effects of hysteresis and constitutive nonlinearities. To determine $W_{d}$ when $d$ consists of the error accrued when approximating the inverse filter as depicted in Figure 10b, a signal having the same frequency as

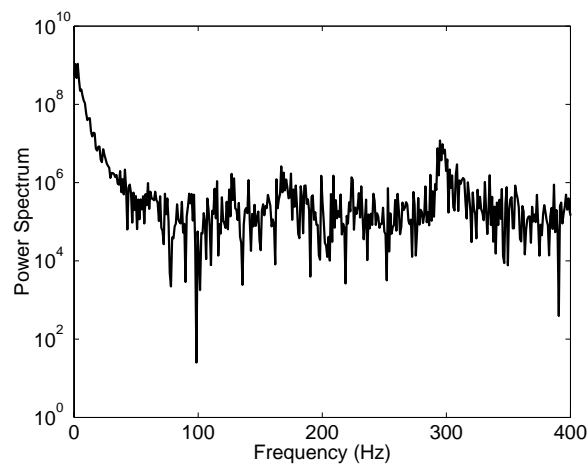

(a)

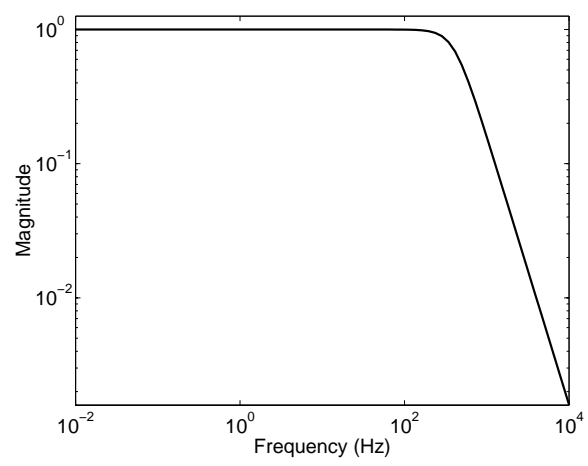

(b)

Figure 13: (a) Power spectrum of $d$, and (b) frequency response of $W_{d}$ for the disturbance $d$ due to inverse filtering error. 


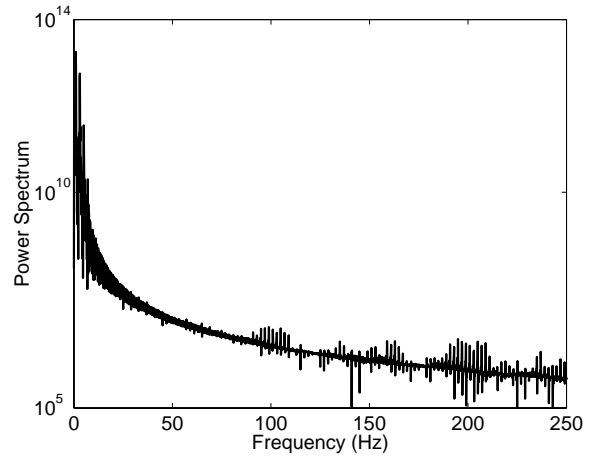

(a)

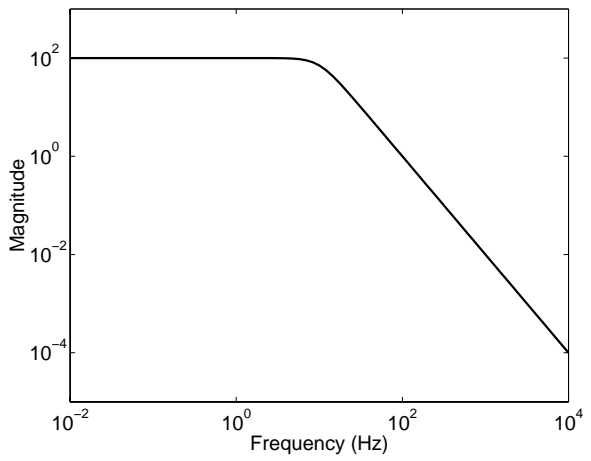

(b)

Figure 14: (a) Power spectrum of $d$, and (b) frequency response of $W_{d}$ for the disturbance $d$ due to scaled but uncompensated hysteresis and nonlinearities.

the reference signal was applied to the approximate inverse filter and the result was fed into the energy-based hysteresis model. The power spectrum of the output $d$ from the hysteresis model was subsequently analyzed to determine $W_{d}$. From the power spectrum plotted in Figure 13a, it can be concluded that in this case $W_{d}$ should weight the strong low frequency component as well as measured frequencies around $350 \mathrm{~Hz}$ so it was specified as a lowpass Butterworth filter having a cutoff frequency of $400 \mathrm{~Hz}$ as illustrated in Figure 13b. Higher order filters can be used if a steeper rolloff outside the frequency band is desired but the price paid for increased order is an increased number of states in the open loop state space system representation.

In the second case, a linear filter was employed to eliminate scaling differences between field and magnetization values, but no inverse filter was employed so the disturbance $d$ includes the scaled hysteresis and constitutive nonlinearities. The power spectrum of a signal fed through the resulting system is plotted in Figure 14 along with the frequency response of a fourth-order lowpass Butterworth filter having a cutoff frequency of $10 \mathrm{~Hz}$. A comparison between this power spectrum and that in Figure 13 for the approximate inverse reveals two items: the energy content is significantly higher and the frequency spectrum lower when the disturbance consists of uncompensated hysteresis and nonlinearities than when it is comprised of inversion errors. As will be illustrated in the examples of Section 4, this significantly impacts the tracking authority of robust control designs employing the two strategies and limits the accuracy of designs which accommodate the uncompensated hysteresis and constitutive nonlinearities solely as disturbances.

The weighting function on the error signal is specified as $W_{e}=\frac{\gamma_{e}}{s+\epsilon_{e}}$ with $\gamma_{e}=1.8 \times 10^{6}$ and $\epsilon_{e}=1 \times 10^{-8}$. An integrator is chosen to prevent the error from achieving steady state at a nonzero value and the pole was shifted slightly off zero to ensure that the controller is realizable. Finally, the weighting function on the controller output was taken to be $W_{u}=5 \times 10^{-6}$. Since we do not experience problems with saturation or overly large currents to the solenoid, we minimally weight $\widehat{u}$ to focus the control effort on tracking and disturbance rejection. Note that the functions $W_{e}$ and $W_{u}$ satisfy the requirement placed on them in Section 3.1, that is, $D=0$ in the state space realization of $W_{e}$ and $D=5 \times 10^{-6}$ in the realization of $W_{u}$.

\subsection{Robust Control Theory}

The transfer function matrix (22) provides a representation for the transducer system, including weighting filters, whereas the formulation (23) quantifies the state space representation associated with the transfer functions from inputs to outputs. We now specify control laws used to compute the gains $K$ by minimizing specific norms of the closed loop system representation $T$. We consider two measures of robust performance, namely, the $\mathcal{H}_{2}$ norm

$$
\|T\|_{2}^{2}=\frac{1}{2 \pi} \int_{-\infty}^{\infty} \operatorname{trace}\left[T^{*}(i \omega) T(i \omega)\right] d \omega
$$

and the $\mathcal{H}_{\infty}$ norm

$$
\|T\|_{\infty}=\sup _{\omega \in \mathbb{R}} \bar{\sigma}[T(j \omega)]
$$


where $\bar{\sigma}[T(j \omega)]$ denotes the maximal singular values of the closed loop map $T$. Since the sensor noise and disturbances due to approximation errors in the inverse filters or uncompensated hysteresis are included as inputs to the system depicted in Figure 11, the robust control laws will be designed to minimize the norms of the maps from these inputs to the system outputs; that is, from $w$ to $\widehat{e}$ and $w$ to $\widehat{u}$.

\subsection{1 $\mathcal{H}_{2}$ Optimal Control Design}

Employing the notation defined in (23) and (24), the optimal $\mathcal{H}_{2}$ control formulation incorporates two Hamiltonian matrices

$$
H_{2}=\left[\begin{array}{cc}
A-B_{2} R_{1}^{-1} D_{12}^{*} C_{1} & -B_{2} R_{1}^{-1} B_{2}^{*} \\
-C_{1}^{*}\left(I-D_{12} R_{1}^{-1} D_{12}^{*}\right) C_{1} & -\left(A-B_{2} R_{1}^{-1} D_{12}^{*} C_{1}\right)^{*}
\end{array}\right]
$$

and

$$
J_{2}=\left[\begin{array}{cc}
\left(A-B_{2} R_{1}^{-1} D_{12}^{*} C_{1}\right)^{*} & -C_{2}^{*} R_{2}^{-1} C_{2} \\
-B_{1}\left(I-D_{21}^{*} R_{2}^{-1} D_{21}\right) B_{1}^{*} & -\left(A-B_{2} R_{1}^{-1} D_{12}^{*} C_{1}\right)
\end{array}\right],
$$

where $R_{1} \equiv D_{12}^{*} D_{12}>0$ and $R_{2} \equiv D_{21} D_{21}^{*}>0$, along with the Riccati equations

$$
\begin{aligned}
& \left(A-B_{2} R_{1}^{-1} D_{12}^{*} C_{1}\right)^{*} X_{2}+X_{2}\left(A-B_{2} R_{1}^{-1} D_{12}^{*} C_{1}\right)+X_{2}\left(-B_{2} R_{1}^{-1} B_{2}^{*}\right) X_{2}-C_{1}^{*}\left(I-D_{12} R_{1}^{-1} D_{12}^{*}\right) C_{1}=0 \\
& \left(A-B_{2} R_{1}^{-1} D_{12}^{*} C_{1}\right) Y_{2}+Y_{2}\left(A-B_{2} R_{1}^{-1} D_{12}^{*} C_{1}\right)^{*}+Y_{2}\left(-C_{2}^{*} R_{2}^{-1} C_{2}\right) Y_{2}-B_{1}\left(I-D_{21}^{*} R_{2}^{-1} D_{21}\right) B_{1}^{*}=0 .
\end{aligned}
$$

The following theorem from [41] provides conditions guaranteeing the existence of a unique $\mathcal{H}_{2}$ feedback gain.

Theorem 1: There exists a unique controller which minimizes the $\mathcal{H}_{2}$ norm of the closed loop system if

1. $H_{2} \in \operatorname{dom}(\operatorname{Ric})$ and $X_{2} \equiv \operatorname{Ric}\left(H_{2}\right)>0$

2. $J_{2} \in \operatorname{dom}(\operatorname{Ric})$ and $Y_{2} \equiv \operatorname{Ric}\left(J_{2}\right)>0$.

The optimal $\mathcal{H}_{2}$ control gain is given by

$$
K \equiv\left[\begin{array}{c|c}
A_{2} & -L_{2} \\
\hline F_{2} & 0
\end{array}\right]
$$

where

$$
A_{2}=A+B_{2} F_{2}+L_{2} C_{2} \quad, \quad F_{2}=-R_{1}^{-1}\left(B_{2}^{*} X_{2}+D_{12}^{*} C_{1}\right) \quad, \quad L_{2}=-\left(Y_{2} C_{2}^{*}+B_{1} D_{21}^{*}\right) R_{2}^{-1} .
$$

We note that Assumptions A2, A3 and A4 from Section 3.1.1 guarantee that $H_{2} \in \operatorname{dom}($ Ric) whereas Assumptions $\mathrm{A} 1, \mathrm{~A} 3$ and $\mathrm{A} 5$ guarantee that $J_{2} \in \operatorname{dom}(\mathrm{Ric})$.

\subsection{2 $\mathcal{H}_{\infty}$ Sub-optimal Control Design}

Secondly, we consider robust control design utilizing the $\mathcal{H}_{\infty}$ norm defined in (26). As detailed in [41], the formulation of a suboptimal $\mathcal{H}_{\infty}$ control law which guarantees $\|T\|_{\infty}<\gamma, \gamma>0$, yields the Hamiltonian matrices

$$
H_{\infty}=\left[\begin{array}{cc}
A & \gamma^{-2} B_{1} B_{1}^{*}-B_{2} B_{2}^{*} \\
-C_{1}^{*} C_{1} & -A^{*}
\end{array}\right]
$$

and

$$
J_{\infty}=\left[\begin{array}{cc}
A^{*} & \gamma^{-2} C_{1}^{*} C_{1}-C_{2}^{*} C_{2} \\
-B_{1} B_{1}^{*} & -A
\end{array}\right]
$$

along with the Riccati equations

$$
\begin{aligned}
& A^{*} X_{\infty}+X_{\infty} A+X_{\infty}\left(\gamma^{-2} B_{1} B_{1}^{*}-B_{2} B_{2}^{*}\right) X_{\infty}+C_{1}^{*} C_{1}=0 \\
& A Y_{\infty}+Y_{\infty} A^{*}+Y_{\infty}\left(\gamma^{-2} C_{1}^{*} C_{1}-C_{2}^{*} C_{2}\right) Y_{\infty}+B_{1} B_{1}^{*}=0 .
\end{aligned}
$$


The primary difference between the Hamiltonian matrices arising in the $\mathcal{H}_{\infty}$ formulation and the Hamiltonian matrices for the $\mathcal{H}_{2}$ formulation is that the $(1,2)$ blocks of $H_{\infty}$ and $J_{\infty}$ are not sign-definite. Therefore, a solution to the Riccati equations can not be guaranteed for all $\gamma$. We note that in the limit $\gamma \rightarrow \infty$, the $\mathcal{H}_{\infty}$ Hamiltonians (30) and (31) converge to the $\mathcal{H}_{2}$ Hamiltonians (27) and (28).

The following theorem from [41] guarantees the existence of an $\mathcal{H}_{\infty}$ sub-optimal control law.

Theorem 2: There exists an admissible controller such that $\|T\|_{\infty}<\gamma$ if and only if

1. $H_{\infty} \in \operatorname{dom}($ Ric $)$ and $X_{\infty} \equiv \operatorname{Ric}\left(H_{\infty}\right)>0$

2. $J_{\infty} \in \operatorname{dom}(\operatorname{Ric})$ and $Y_{\infty} \equiv \operatorname{Ric}\left(J_{\infty}\right)>0$

3. $\rho\left(X_{\infty} Y_{\infty}\right)<\gamma^{2}$.

The $\mathcal{H}_{\infty}$ suboptimal control law is given by

$$
K \equiv\left[\begin{array}{c|c}
A_{\infty} & -Z_{\infty} L_{\infty} \\
\hline F_{\infty} & 0
\end{array}\right]
$$

where

$$
A_{\infty}=A+\gamma^{-2} B_{1} B_{1}^{*} X_{\infty}+B_{2} F_{\infty}+Z_{\infty} L_{\infty} C_{2} \quad, \quad F_{\infty}=-B_{2}^{*} X_{\infty} \quad, \quad L_{\infty}=-Y_{\infty} C_{2}^{*} .
$$

While the control input provided by the system (32) is suboptimal in the sense that it provides a closed loop system with an $\mathcal{H}_{\infty}$ norm less than $\gamma$, the Matlab routine hinfsyn can be utilized to decrease $\gamma$ until an assumption of Theorem 2 is violated. This provides control inputs which are nearly optimal.

\section{Numerical Examples}

We illustrate here the performance of the $\mathcal{H}_{2}$ and $\mathcal{H}_{\infty}$ control designs in the context of the motivating milling application. As illustrated in Figure 1, a magnetostrictive transducer is used to position a cutting head adjacent to the unmilled object. During the subsequent periodic milling process, it is required that the transducer maintain tolerances of 1-2 $\mu \mathrm{m}$ while operating in hysteretic and nonlinear regimes to achieve the required stroke and force inputs. The ODE model (21) is used to quantify the cutting head position with hysteresis and constitutive nonlinearities characterized by the discrete magnetization model (14). The inverse filter is constructed via Algorithm 1 in Section 2.2 with $\Delta H$ chosen to be sufficiently large to permit eventual real-time implementation which also serves to illustrate the performance of the control algorithms with nontrivial disturbances due to inversion errors. For all simulations, we employed the parameters specified in the validation example of Section 2.1 which yielded the model fits to experimental data plotted in Figure 6. Finally, as noted in Section 3.1.1, a minimal realization having 31 states was employed in the simulations.

The physical milling device is designed to operate at $3000 \mathrm{rpm}$ which will introduce eddy current losses in general transducer designs. To focus the discussion on control issues associated with the inherently nonlinear and hysteretic transducers, we neglect this source of losses which is appropriate for magnetostrictive transducers utilizing laminated Terfenol rods and casings to minimize currents. In this case, it is appropriate to normalize the reference frequency to $1 \mathrm{~Hz}$. The extensions required to accommodate eddy current losses for general transducer designs will entail the incorporation of additional physical mechanisms in the magnetization models but no changes in the robust control designs employed here.

For the $\mathcal{H}_{2}$ and $\mathcal{H}_{\infty}$ designs, we consider three cases. In all three cases, a $60 \mathrm{~Hz}$ signal $s$ having a magnitude of $5 \times 10^{-6} \mathrm{~m}$ was added to the simulated tip displacement $y$ to illustrate the effects of narrowband sensor noise. The same noise signal was added in all simulations and because the magnitude of the reference trajectory is $1 \times 10^{-4}$, this represents a $5 \%$ noise to data ratio. Similar results were obtained when low magnitude, high frequency noise $n$ was added but to simplify the discussion, we omit this source of noise in the examples. To provide a baseline for comparison, we employed a linear input relation in the first case to illustrate the performance of the control laws in the absence of a disturbance $d$. This can also be interpreted as the case when the inverse filter perfectly compensates for the hysteresis and constitutive nonlinearities. Secondly, we 
employ the approximate inverse filter so that the disturbance $d$ consists of discretization errors accrued when employing a large stepsize $\Delta H$. In the final case, we consider robust control design when a linear filter is used to provide the same scale in the field and magnetization, as illustrated in Figure 10c, but no inverse filter is employed. This illustrates the authority provided by the robust control designs which treat the uncompensated hysteresis and nonlinearities as the disturbance $d$. We emphasize that in each case, we optimized both the filter design and choice of control weights to achieve optimal performance for the respective disturbances.

\section{1 $\mathcal{H}_{2}$ Control Design}

We consider first the tracking authority achieved using the $\mathcal{H}_{2}$ design summarized in Section 3.3 .1 for the three disturbance cases. The same $5 \%$ sensor noise signal is applied in all three examples.

\section{Case i: Sensor Noise $s$ but No Disturbance}

The tracking authority obtained in the presence of $60 \mathrm{~Hz}$ sensor noise with a noise-to-data ratio of $5 \%$ is illustrated in Figure 15a with the corresponding tracking error $e=r-y$ plotted in Figure 15b. The presence of the sensor noise can be observed in the two flat portions of the reference signal $r$ which corresponds to the times when the cutting head is held fixed. Following a slight spike when the milling commences, the error is maintained well within the tolerance of $2 \mu \mathrm{m}$ and has a magnitude of $1 \mu \mathrm{m}$ after 2.5 seconds. We note that because the accuracy during the milling process was deemed more important than the accuracy during the positioning and holding periods, the filter $W_{r}$ described in Section 3.2 was chosen to weight the $1 \mathrm{~Hz}$ signal at the slight expense of quasistatic regimes. This can of course be modified if tolerances during other phases of the milling process are considered more crucial. These results represent the baseline tracking achievable for a linear transducer or when inverse filtering has no error.

\section{Case ii: Sensor Noise $s$ and Disturbance $d$ Due to Inversion Error}

Secondly, we consider the $\mathcal{H}_{2}$ design obtained using the approximate inverse filter specified by Algorithm 1. The disturbance $d$ in this case consists of discretization error for the filter which has the frequency profile depicted in Figure 13a. The weight $W_{d}$ was taken to be the lowpass Butterworth filter depicted in Figure 13b.

The computed tip position $y$ and reference trajectory $r$, along with the tracking error $e$, are plotted in Figure 16. The $H-M$ and $H-y$ relations are plotted in Figure 17 to illustrate the nonlinear and hysteretic relationship between the applied field $H$, magnetization $M$ and tip displacement - these relations also illustrate the necessity of employing hysteresis models which guarantee the closure of biased minor loops. We note that the $H-M$ and $H-y$ relations are qualitatively similar due to the assumption of a linear relation between $M$ and $y$ made when constructing the transducer model (21) for biased operating regimes. The effect of the bias is readily noted if one compares Figure 17 with the unbiased validation results in Figure 6.

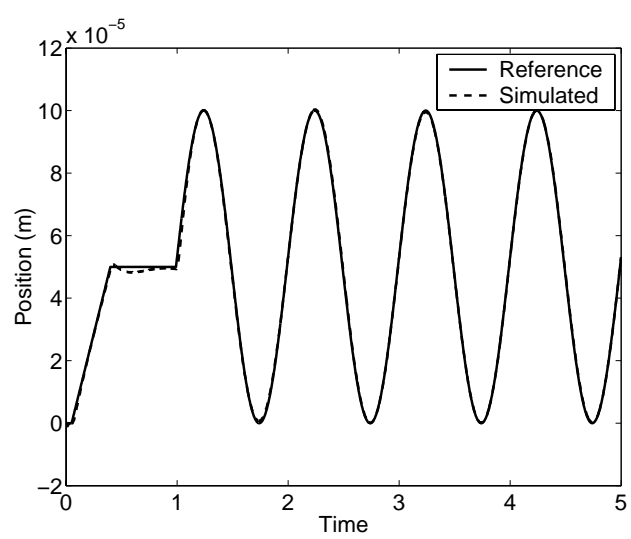

(a)

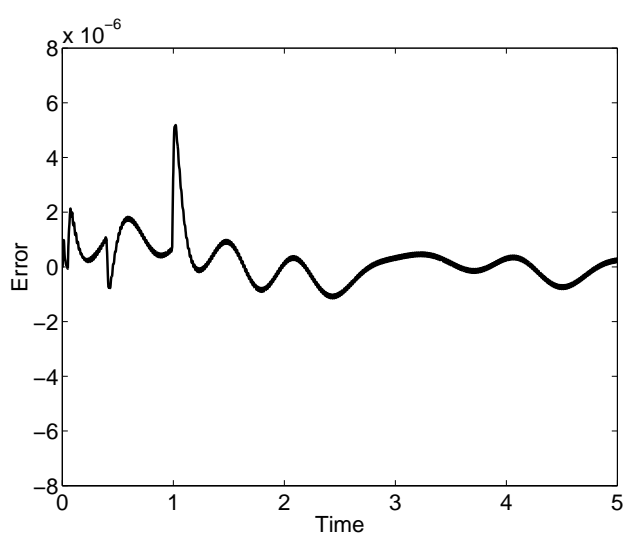

(b)

Figure 15: $\mathcal{H}_{2}$ design incorporating sensor noise $s$ but no disturbance $d$. (a) Reference trajectory and simulated cutting head position. (b) Error in cutting head position. 


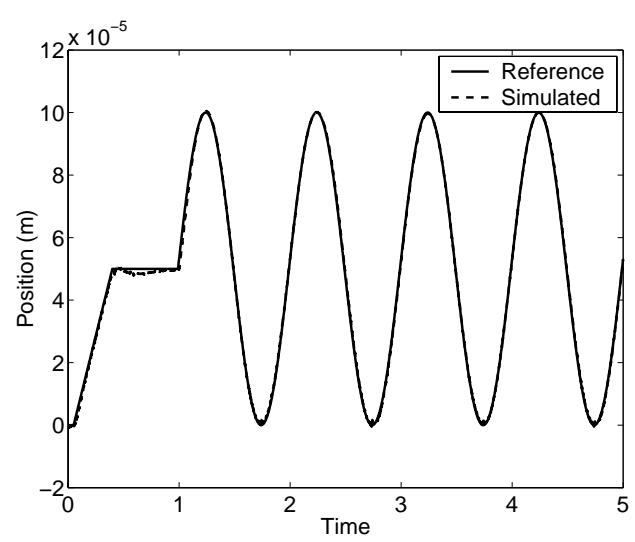

(a)

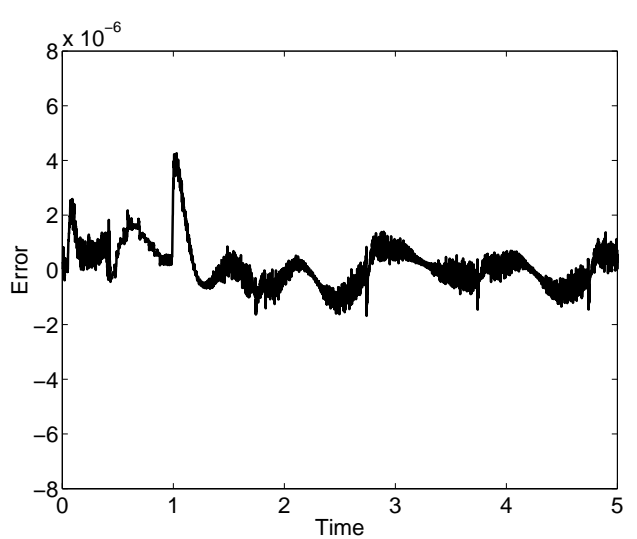

(b)

Figure 16: $\mathcal{H}_{2}$ design incorporating sensor noise $s$ and the disturbance $d$ due to discretization errors in the inverse filter. (a) Reference trajectory and simulated cutting head position. (b) Error in cutting head position.

The transducer starts at a position of $y=0$ for $H=0$, and following slightly negative excursions due to sensor noise, a field of $2 \times 10^{4} \mathrm{~A} / \mathrm{m}$ is applied to position the head adjacent to the ingot. It is observed that a decrease in field is required to counter the slight overshoot and sensor noise before the periodic milling process commences. During the milling process, the error is maintained within the tolerance level of $2 \mu \mathrm{m}$ with the largest errors occurring at field reversal.

To further illustrate robustness properties of the $\mathcal{H}_{2}$ design, we formulate the tracking error

$$
\begin{aligned}
e & =r-y \\
& =r-(n+s+P[d+K[e]]) \\
& =S[r]-S[n]-S[s]-S_{d}[d]
\end{aligned}
$$

in terms of the sensitivity function

$$
S=(I+P K)^{-1},
$$

which is the transfer matrix from $r, n$ and $s$ to $e$, and the disturbance sensitivity function

$$
S_{d}=(I+P K)^{-1} P,
$$

which maps $d$ to $e$. The frequency responses of $S$ and $S_{d}$ illustrate the robustness of the design to certain frequency inputs.

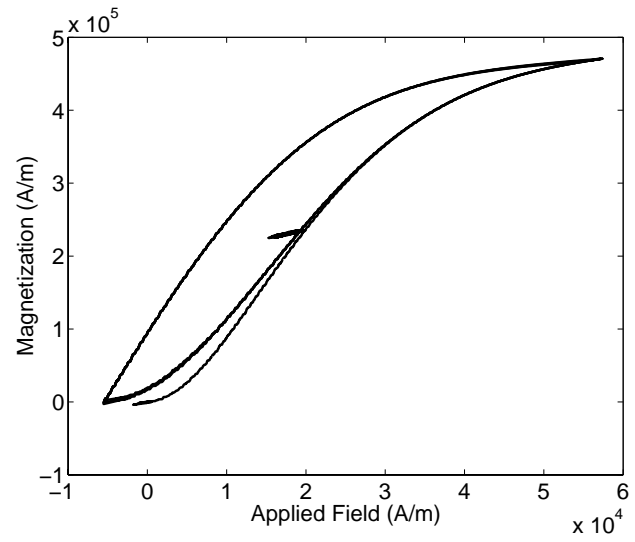

(a)

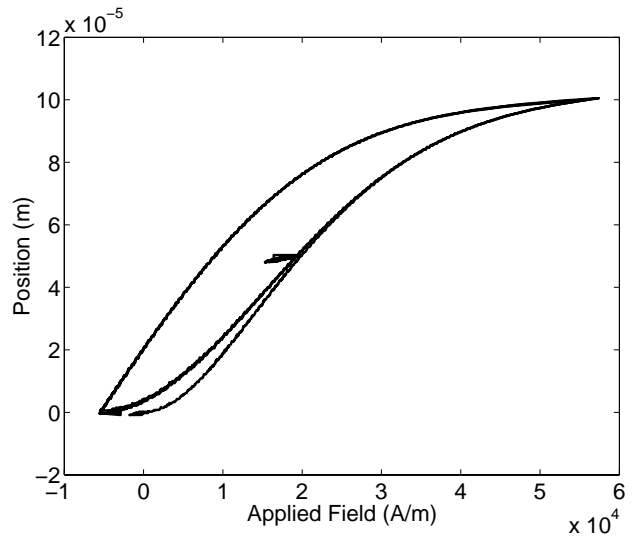

(b)

Figure 17: (a) Hysteretic relation between the field $H$ and magnetization $M$, and (b) relation between $H$ and the displacement $y$. 


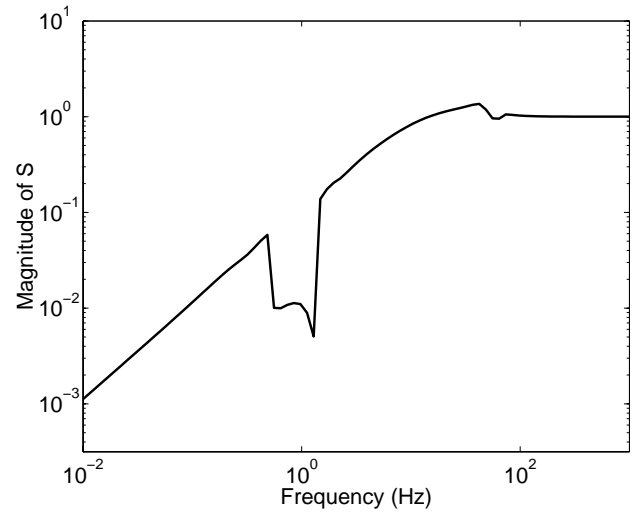

(a)

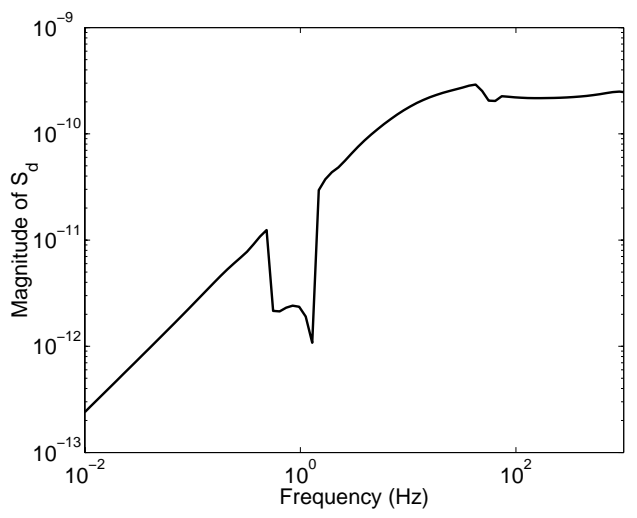

(b)

Figure 18: Frequency response of (a) the $\mathcal{H}_{2}$ sensitivity function $S$, and (b) disturbance sensitivity function $S_{d}$ for the disturbance $d$ due to discretization errors in the inverse filter.

For the disturbance $d$ consisting of inversion error, the frequency responses of $S$ and $S_{d}$ are plotted in Figure 18. Both functions exhibit a strong dip in magnitude in the neighborhood of $1 \mathrm{~Hz}$ dictated by the tracking filter $W_{r}$ which provides the method with robust tracking authority for the reference signal. The dip below a magnitude of 1 at $60 \mathrm{~Hz}$ reflects the rejection of sensor noise $s$ using the filter $W_{s}$. Finally, the disturbance function $S_{d}$ exhibits strong attenuation throughout the interval $[0,350 \mathrm{~Hz}]$ thus illustrating that the high frequency disturbance due to inversion error is being adequately accommodated. Hence the $\mathcal{H}_{2}$ design utilizing the approximate inverse filter achieves the specified tracking criteria.

\section{Case iii: Sensor Noise $s$ and Disturbance $d$ Due to Uncompensated Hysteresis and Nonlinearities}

Finally, we consider the effectiveness of the $\mathcal{H}_{2}$ design obtained using a linear filter to provide the same scale between field and magnetization values but no compensation for hysteresis or constitutive nonlinearities. The resulting disturbance has the frequency profile plotted in Figure 14a and the filter $W_{d}$ depicted in Figure 14b was employed in the $\mathcal{H}_{2}$ design.

The tracking performance and error $e$, while operating in hysteretic regimes qualitatively similar to those depicted in Figure 17, are plotted in Figure 19. It is observed that in this case, significant phase delays are present at the nonlinear high drive levels which produces errors of between 6 and $7 \mu \mathrm{m}$. The errors of 3 to $4 \mu \mathrm{m}$ as fields are cycled to zero also exceed the $2 \mu \mathrm{m}$ tolerance. Hence the optimal $\mathcal{H}_{2}$ design in this case

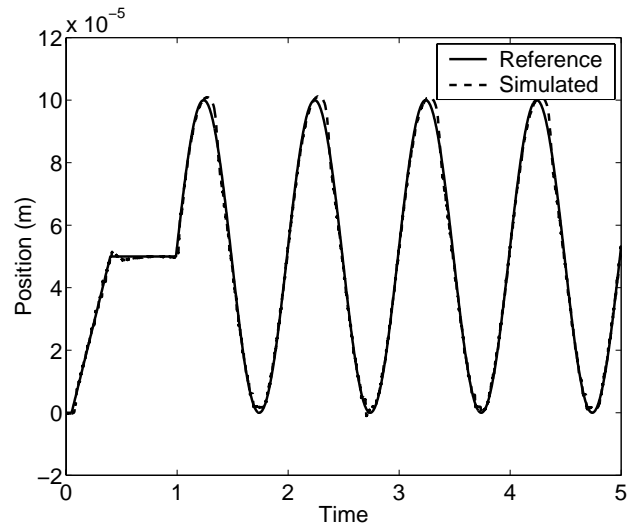

(a)

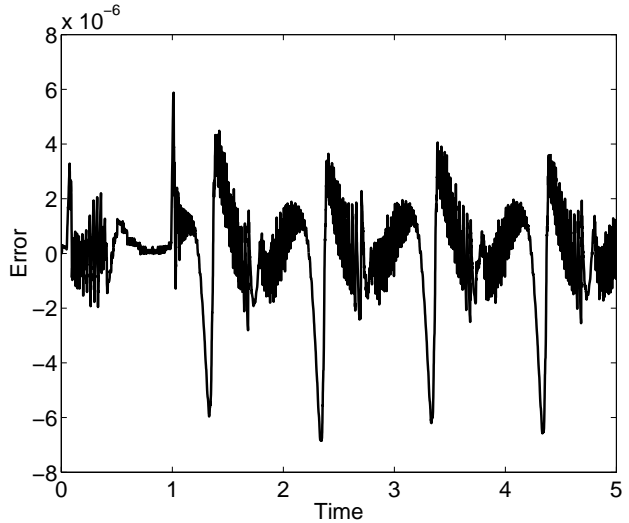

(b)

Figure 19: $\mathcal{H}_{2}$ design incorporating sensor noise $s$ and the disturbance $d$ due to uncompensated hysteresis and constitutive nonlinearities. (a) Reference trajectory and simulated cutting head position. (b) Error in cutting head position. 
does not achieve the control criteria since it cannot fully accommodate the uncompensated hysteresis and constitutive nonlinearities. This illustrates the necessity of employing the approximate inverse filter to achieve the stringent tracking tolerances dictated by the application.

\section{$4.2 \mathcal{H}_{\infty}$ Control Design}

We illustrate here the performance of the $\mathcal{H}_{\infty}$ design summarized in Section 3.3.2 in the absence of a disturbance $d$, and for disturbances comprised of inversion errors and uncompensated hysteresis and constitutive nonlinearities. In all cases, we incorporate $60 \mathrm{~Hz}$ sensor noise $s$ having a noise-to-data ratio of $5 \%$.

\section{Case i: Sensor Noise $s$ but No Disturbance}

To provide a baseline for comparison, we consider first the tracking accuracy obtained in the presence of sensor noise $s$ but no disturbance $d$. The tracking capability provided by the $\mathcal{H}_{\infty}$ design under these conditions is illustrated in Figure 20. It is observed that during the holding period, the control maintains a tolerance of 1-2 $\mu \mathrm{m}$ and then tracks to within $1 \mu \mathrm{m}$ during the periodic milling process. Hence the $\mathcal{H}_{\infty}$ design achieves the control specifications in the absence of a disturbance $d$ which represents either perfect inverse compensation or linear transducer behavior. A comparison between Figures 20 and 15 indicates nearly identical tracking performance for the $\mathcal{H}_{\infty}$ and $\mathcal{H}_{2}$ designs in this control regime.

\section{Case ii: Sensor Noise $s$ and Disturbance $d$ Due to Inversion Error}

We next employ Algorithm 1 to construct an approximate inverse filter so that $d$ consists of inversion errors having the frequency profile depicted in Figure 13a. The simulated tip position and tracking error for the $\mathcal{H}_{\infty}$ control designed for this disturbance are plotted in Figure 21 and the frequency responses of the sensitivity function $S$ and disturbance sensitivity function $S_{d}$, respectively defined in (34) and (35), are plotted in Figure 22. The hysteretic relationship between the applied field $H$ and tip displacement $y$ is sufficiently similar to the $\mathcal{H}_{2}$ phase plot depicted in Figure 17 that we reference that figure when discussing the $\mathcal{H}_{\infty}$ tracking performance.

Like the $\mathcal{H}_{2}$ design, the $\mathcal{H}_{\infty}$ maintains a tolerance of under $2 \mu \mathrm{m}$ during the periodic milling phase thus achieving the control objectives. The frequency responses of $S$ and $S_{d}$ also reflect diminished magnitudes at $1 \mathrm{~Hz}$ and $60 \mathrm{~Hz}$ due to the lowpass and narrowband filters, and the magnitude of $S$ is maintained below 1 at higher frequencies so that high frequency disturbances are not being amplified.

A comparison of Figure 21 with Figure 16 and Figure 22 with Figure 18 illustrates that while the $\mathcal{H}_{\infty}$ and $\mathcal{H}_{2}$ designs exhibit minor differences in tracking authority and robustness, both yield similar qualitative results. Because the $\mathcal{H}_{2}$ design is more efficient to implement, it is somewhat advantageous in this regime from a technological perspective.

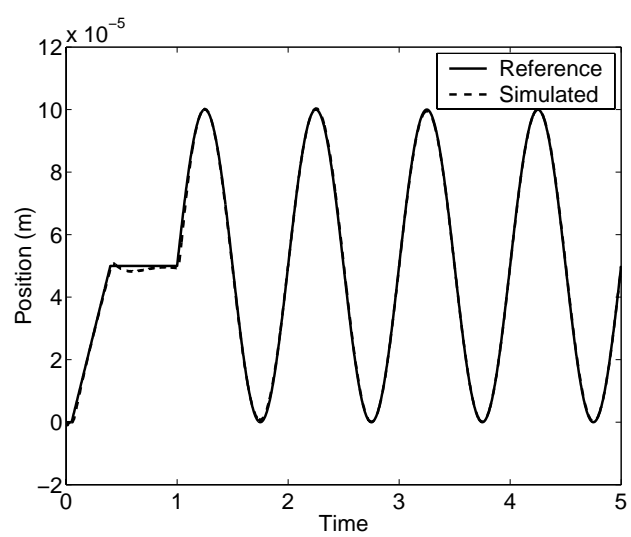

(a)

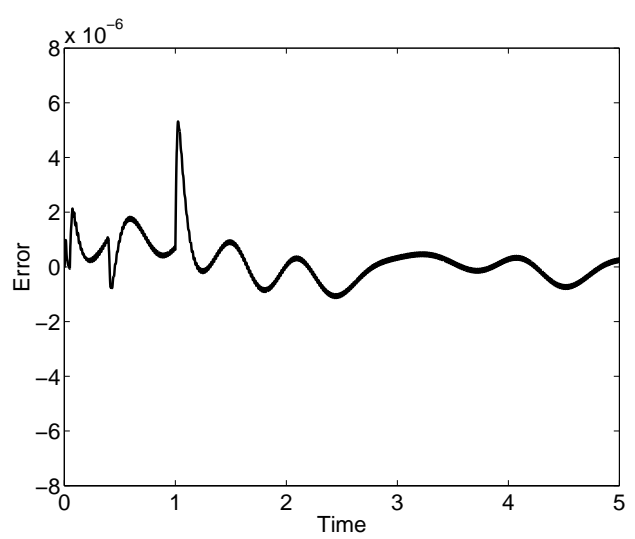

(b)

Figure 20: $\mathcal{H}_{\infty}$ design incorporating sensor noise $s$ but no disturbance $d$. (a) Reference trajectory and simulated cutting head position. (b) Error in cutting head position. 


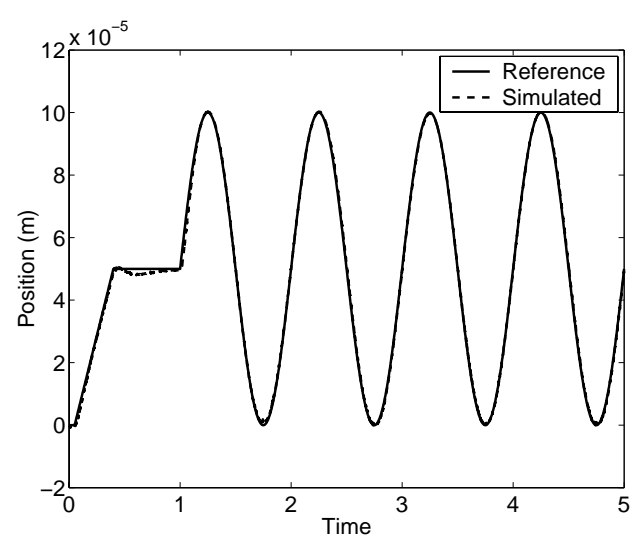

(a)

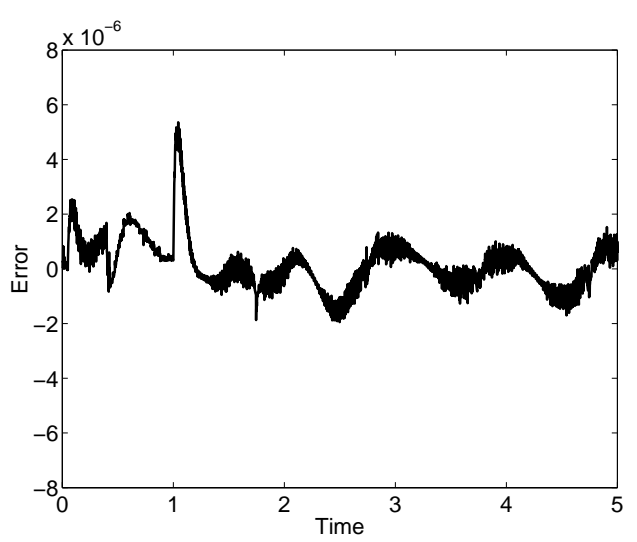

(b)

Figure 21: $\mathcal{H}_{\infty}$ design incorporating sensor noise $s$ and the disturbance $d$ due to discretization errors in the inverse filter. (a) Reference trajectory and simulated cutting head position. (b) Error in cutting head position.

\section{Case iii: Sensor Noise $s$ and Disturbance $d$ Due to Uncompensated Hysteresis and Nonlinearities}

In the final case, the disturbance consists of scaled but uncompensated hysteresis and constitutive nonlinearities having the frequency profile plotted in Figure 14a. The $\mathcal{H}_{\infty}$ design using the filter $W_{d}$ depicted in Figure 14b yielded the tip displacements and tracking errors plotted in Figure 23. These results exhibit the same tendency observed in Figure 19 for the $\mathcal{H}_{2}$ design in the sense that hysteresis-induced phase delays produce tracking errors on the order of 6-7 $\mu \mathrm{m}$ in the nonlinear, high drive regimes required to complete the strokes at the apogee of the cycle. For these disturbances, neither the $\mathcal{H}_{\infty}$ nor $\mathcal{H}_{2}$ designs provided the authority required to fully compensate for the hysteresis and achieve the specified tolerances of 1-2 $\mu \mathrm{m}$.

\section{Concluding Remarks}

This paper addresses issues associated with robust control design for high performance actuators operating in nonlinear and hysteretic regimes and illustrates that in some cases, the incorporation of inverse filters to reduce the severity of unmodeled disturbances is required to achieve stringent tracking tolerances. A fundamental component in these robust control designs is the development of nonlinear hysteresis models which accurately characterize transducer behavior and permit the construction of approximate model inverses which are sufficiently efficient to permit eventual real-time implementation. The construction of such a modeling

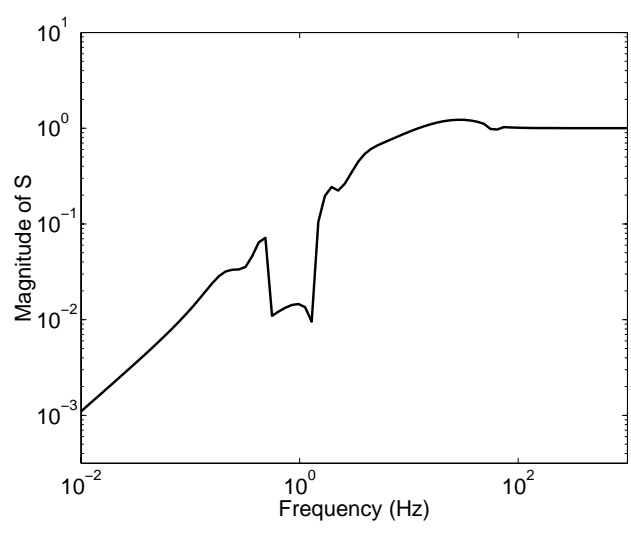

(a)

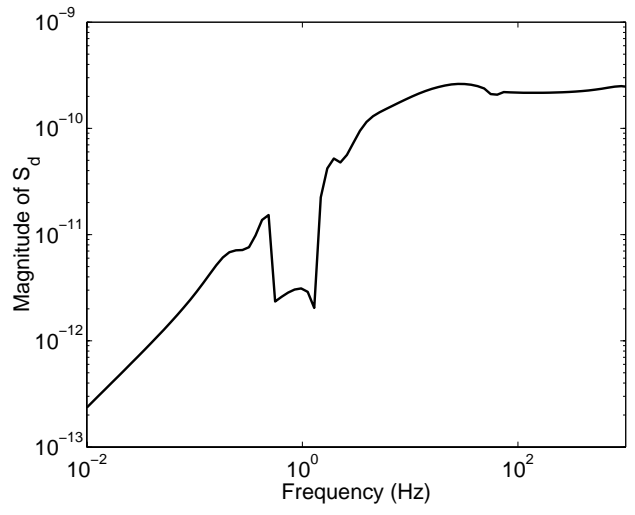

(b)

Figure 22: (a) $\mathcal{H}_{\infty}$ sensitivity function, and (b) disturbance sensitivity function for the disturbance $d$ due to discretization errors in the inverse filter. 


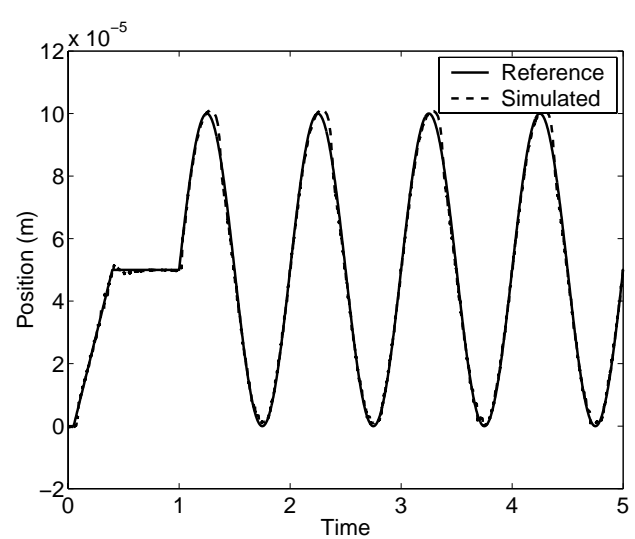

(a)

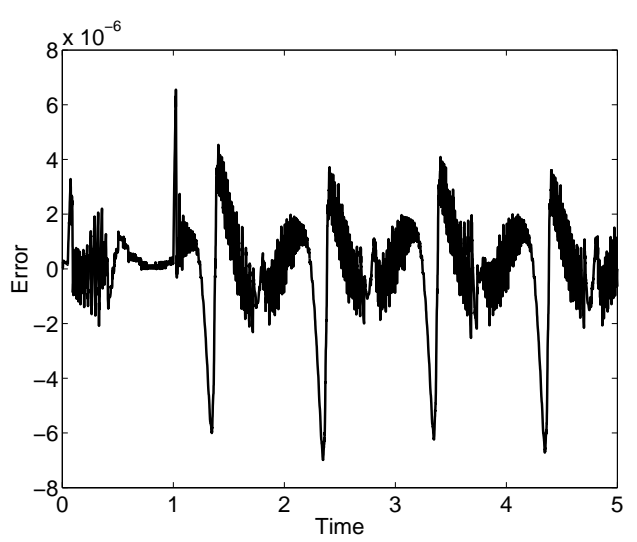

(b)

Figure 23: $\mathcal{H}_{\infty}$ design incorporating sensor noise $s$ and the disturbance $d$ due to uncompensated hysteresis and constitutive nonlinearities. (a) Reference trajectory and simulated cutting head position. (b) Error in cutting head position.

framework is illustrated in the context of a magnetostrictive transducer employed for high accuracy milling. Furthermore, it is illustrated in $[35,36]$ that this framework can also be employed to quantify the hysteretic and nonlinear behavior of ferroelectric and ferroelastic compounds, including piezoceramic and shape memory alloys, so it provides a unified framework for constructing inverse-based robust control designs for a broad range of presently employed smart material transducers.

To accommodate a variety of disturbances, we considered both $\mathcal{H}_{2}$ and $\mathcal{H}_{\infty}$ designs. To provide a baseline for comparison, we first considered the tracking accuracy obtained in the presence of $60 \mathrm{~Hz}$ sensor noise having a noise-to-data ratio of $5 \%$ but no disturbances due to actuator nonlinearities. In this regime, we illustrated through numerical examples that both the $\mathcal{H}_{2}$ and $\mathcal{H}_{\infty}$ designs easily maintain the specified tolerances of 1-2 $\mu \mathrm{m}$ during the motivating periodic milling process. Secondly, we considered the performance of robust designs utilizing an approximate model-based inverse filter with highpass filters constructed to accommodate the disturbance due to inversion errors. While the tracking accuracy in this case was slightly diminished from that obtained with no disturbance, both the $\mathcal{H}_{2}$ and $\mathcal{H}_{\infty}$ designs maintained tracking tolerances under $2 \mu \mathrm{m}$ during the milling process and hence achieved the control objectives. The third design utilized a linear factor to provide the same scales between field and magnetization but treated the scaled hysteresis and constitutive nonlinearities as disturbances to be accommodated by the robust control laws. In this case, both the $\mathcal{H}_{2}$ and $\mathcal{H}_{\infty}$ designs exhibited tracking errors on the order of 6-7 $\mu \mathrm{m}$ due to hysteresis-induced phase delays and unaccommodated nonlinearities - hence neither design is able to achieve the stringent tracking tolerance when the entire impetus for attenuating hysteresis and nonlinearities is placed on the control law.

We note that the use of the scale factor is advantageous for ferromagnetic transducer but is not crucial when employing SI (Sommerfield) units since field and magnetization levels often differ by only 1-2 orders of magnitude. It will be much more crucial when designing robust control algorithms for piezoceramic materials in the absence of inverse compensation since electric fields often have magnitudes on the order of $10^{6} \mathrm{~V} / \mathrm{m}$ whereas corresponding polarization levels have values on the order of $0.1-0.3 \mathrm{C} / \mathrm{m}^{2}$.

A crucial component of the control design involves the construction of filters which weight the reference signal, errors, sensor noise, and disturbances. For the milling example considered here, the choice of appropriate weighting filters had more impact on the final tracking authority than did the specification of $\mathcal{H}_{2}$ versus $\mathcal{H}_{\infty}$ control designs.

The necessity of considering inverse filter-based designs will be augmented as operating frequencies are increased due to increasing noise-to-data ratios, larger measured hysteresis loops, and diminishing authority of highpass filters. The incorporation of the physical mechanisms necessary to accommodate frequencydependence in the unified hysteresis models used to construct the inverse filters for magnetostrictive, piezoceramic and shape memory alloy compounds is under present investigation and will be incorporated in future robust control designs. 
Finally, a guiding factor throughout this analysis is the development of robust control designs which are feasible for eventual experimental implementation. This requirement is increasingly stringent for a number of high frequency applications under consideration which target the broadband capabilities of piezoceramic, relaxor ferroelectric and magnetostrictive transducers - e.g., PZT-based, high speed nanopositioning for realtime tracking of biological processes or magnetostrictive transducer design for high speed industrial processes. The control framework developed here was constructed with these implementation requirements in mind and present investigations are focused on the initial experimental implementation of the methods.

\section{Acknowledgments}

This research was supported in part by the Air Force Office of Scientific Research under the grant AFOSRF49620-01-1-0107 and through the NSF grant CMS-009764.

\section{References}

Note: Center for Research in Scientific Computation Technical Reports can be accessed at the web site http://www.ncsu.edu/crsc/reports.html.

[1] T. Başar and P. Bernhard, $\mathcal{H}_{\infty}$ Optimal Control and Related Minimax Design Problems, Birkhäuser, Boston, 1995.

[2] F.T. Calkins, R.C. Smith and A.B. Flatau, "An Energy-based Hysteresis Model for Magnetostrictive Transducers," IEEE Transactions on Magnetics, 36(2), pp. 429-439, 2000.

[3] D. Croft and S. Devasia, "Vibration compensation for high speed scanning tunneling microscopy," Review of Scientific Instruments, 70(12), pp. 4600-4605, 1999.

[4] J.M Cruz-Hernandez and V. Hayward, "An approach to reduction of hysteresis in smart materials," Proceedings of the 1998 IEEE International Conference on Robotics and Automation, Leuven, Belgium, pp. 1510-1515, 1998.

[5] M.J. Dapino, F.T. Calkins and A.B. Flatau, "Magnetostrictive Devices," Wiley Encyclopedia of Electrical and Electronics Engineering, John G. Webster, Ed., John Wiley and Sons, Inc., Volume 12, pp. 278-305, 1999.

[6] M.J. Dapino, R.C. Smith, L.E. Faidley and A.B. Flatau, "A Coupled Structural-Magnetic Strain and Stress Model for Magnetostrictive Transducers," Journal of Intelligent Material Systems and Structures, 11(2), pp. 134-152, 2000.

[7] M.J. Dapino, R.C. Smith and A.B. Flatau, "A Structural Strain Model for Magnetostrictive Transducers," IEEE Transactions on Magnetics, 36(3), pp. 545-556, 2000.

[8] J.C. Doyle, K. Glover, P. Khargonekar and B.A. Francis (1989), "State space solutions to standard $\mathcal{H}_{2}$ and $\mathcal{H}_{\infty}$ control problems," IEEE Transactions on Automatic Control, 34, pp. 831-847.

[9] M.V. Gandhi and B.S. Thompson, Smart Materials and Structures, Chapman and Hall, New York, 1992.

[10] P. Ge and M. Jouaneh, "Tracking control of a piezoceramic actuator," IEEE Transactions on Control Systems Technology, 4(3), pp. 209-216, 1996.

[11] D. Grant and V. Hayward, "Variable structure control of shape memory alloy actuators," IEEE Control Systems Magazine, 17(3), pp. 80-88, 1997.

[12] D. Jiles, Introduction to Magnetism and Magnetic Materials, Chapman and Hall, New York, 1991.

[13] D.C. Jiles and D.L. Atherton, "Theory of ferromagnetic hysteresis," Magnetism and Magnetic Materials, 61, pp. 48-60, 1986. 
[14] D.K. Lindner, Introduction to Signals and Systems, McGraw-Hill, New York, 1999.

[15] J. Luan and F.C. Lee, "Design of a high frequency switching amplifier for smart material actuators with improved current mode control," PESC '98 Record, 29th Annual Power Electronics Specialists Conference, Vol. 1, pp. 59-64, 1998.

[16] J.A. Main and E. Garcia, "Design impact of piezoelectric actuator nonlinearities," Journal of Guidance, Control, and Dynamics, 20(2), pp. 327-32, 1997.

[17] J.A. Main, E. Garcia and D.V. Newton, "Precision position control of piezoelectric actuators using charge feedback," Journal of Guidance, Control, and Dynamics. 18(5), pp. 1068-73, 1995.

[18] J.E. Massad and R.C. Smith, "A Domain Wall Model for Hysteresis in Ferroelastic Materials," CRSC Technical Report CRSC-TR02-34; Journal of Intelligent Material Systems and Structures, to appear.

[19] J.E. Massad, R.C. Smith and G.P. Carman, "A free energy model for thin-film shape memory alloys," CRSC Technical Report CRSC-TR03-03; Proceedings of the SPIE, Smart Structures and Materials 2003, to appear.

[20] D. McFarlane and K. Glover, "A loop shaping design procedure using $\mathcal{H}_{\infty}$ synthesis," IEEE Transactions on Automatic Control, 37(6), pp. 759-769, 1992.

[21] J. Nealis and R.C. Smith, "Partial Inverse Compensation Techniques for Linear Control Design in Magnetostrictive Transducers," Proceedings of the SPIE, Smart Structures and Materials, 2001, Vol. 4326, pp. 462-473, 2001.

[22] J.B. Restorff, "Magnetostrictive materials and devices," Encyclopedia of Applied Physics, Vol. 9, pp. 229244, 1994.

[23] M.V. Salapaka, H.S. Bergh, J. Lai, A. Majumdar and E. McFarland, "Multimode noise analysis of cantilevers for scanning probe microscopy," Journal of Applied Physics, 81(6), pp. 2480-2487, 1997.

[24] S. Salapaka, A. Sebastian, J.P. Cleveland and M.V. Salapaka, "High bandwidth nano-positioner: A robust control approach," Review of Scientific Instruments, 73(9), pp. 3232-3241, 2002.

[25] G. Schitter, P. Menold, H.F. Knapp, F. Allgower and A. Stemmer, "High performance feedback for fast scanning atomic force microscopes," Review of Scientific Instruments, 72(8), pp. 3320-3327, 2001.

[26] A. Sebastion and S. Salapaka, "Hㅐ ${ }_{\infty}$ loop shaping design for nano-positioning," 2003 American Control Conference, to appear.

[27] S. Seelecke and I. Müller, "Shape memory alloy actuators in smart structures - Modeling and simulation," ASME Applied Mechanics Reviews, to appear.

[28] R.C. Smith, "Inverse compensation for hysteresis in magnetostrictive transducers," Mathematical and Computer Modelling, 33, pp. 285-298, 2001.

[29] R.C. Smith, C. Bouton and R. Zrostlik, "Partial and Full Inverse Compensation for Hysteresis in Smart Material Systems," Proceedings of the 2000 American Control Conference.

[30] R.C. Smith and M.J. Dapino, "A macroscopic free energy theory for ferromagnetic hysteresis," IEEE Transactions on Magnetics, to be submitted.

[31] R.C. Smith, M.J. Dapino and S. Seelecke, "A Free Energy Model for Hysteresis in Magnetostrictive Transducers," Journal of Applied Physics, 93(1), pp. 458-466, 2003.

[32] R.C. Smith and C.L. Hom, "A Domain Wall Theory for Ferroelectric Hysteresis," Journal of Intelligent Material Systems and Structures, 10(3), pp. 195-213, 1999. 
[33] R.C. Smith and J.E. Massad, "A Unified Methodology for Modeling Hysteresis in Ferroelectric, Ferromagnetic and Ferroelastic Materials," Proceedings of DETC'01 the ASME Design Engineering Technical Conference and Computers and Information in Engineering Conference, Vol. 6, Pt. B, pp. 1389-1398, 2001.

[34] R.C. Smith and Z. Ounaies, "A Domain Wall Model for Hysteresis in Piezoelectric Materials," Journal of Intelligent Material Systems and Structures, 11(1), pp. 62-79, 2000.

[35] R.C. Smith, S. Seelecke, M.J. Dapino and Z. Ounaies, "A Unified Model for Hysteresis in Ferroic Materials," CRSC Technical Report CRSC-TR03-13; Proceedings of the SPIE, Smart Structures and Materials 2003, to appear.

[36] R.C. Smith, S. Seelecke, M.J. Dapino and Z. Ounaies, "A unified framework for modeling hysteresis in ferroic materials," ASME Applied Mechanics Reviews, to be submitted.

[37] R.C. Smith, S. Seelecke, Z. Ounaies and J. Smith, "A free energy model for hysteresis in ferroelectric materials," CRSC Technical Report CRSC-TR03-01; Journal of Intelligent Material Systems and Structures, to appear.

[38] R.C. Smith and R. Zrostlik, "Inverse Compensation for Ferromagnetic Hysteresis," CRSC Technical Report CRSC-TR99-28; Proc. 38th IEEE Conf. Dec. and Control, Phoenix, AZ, 1999.

[39] X. Tan, R. Venkataraman and P.S. Krishnaprasad, "Control of hysteresis: Theory and experimental results," Smart Structures and Materials 2001, Modeling, Signal Processing and Control in Smart Structures, SPIE Vol. 4326, pp. 101-112, 2001.

[40] G. Tao and P. V. Kokotović, Adaptive Control of Systems with Actuator and Sensor Nonlinearities, John Wiley and Sons, New Jersey, 1996.

[41] K. Zhou and J.C. Doyle, Essentials of Robust Control, Prentice Hall, New Jersey, 1998.

[42] K. Zhou, J.C. Doyle and K. Glover (1996), Robust and Optimal Control, Prentice-Hall, New Jersey. 